\title{
COHOMOLOGY OF 3-POINTS CONFIGURATION SPACES OF COMPLEX PROJECTIVE SPACES
}

\author{
SAMIA ASHRAF ${ }^{1}$, BARBU BERCEANU $^{2}$
}

\begin{abstract}
We compute the Betti numbers and describe the cohomology algebras of the ordered and unordered configuration spaces of three points in complex projective spaces, including the infinite dimensional case. We also compute these invariants for the configuration spaces of three collinear and non-collinear points.
\end{abstract}

\section{INTRODUCTION}

The ordered configuration space of $n$ points $F(X, n)$ of a topological space $X$ is defined as

$$
F(X, n)=\left\{\left(x_{1}, x_{2}, \ldots, x_{n}\right) \in X^{n} \mid x_{i} \neq x_{j} \text { for } i \neq j\right\} .
$$

The symmetric group on $n$ letters acts freely on this space and its orbit space is the unordered configuration space, denoted by $C(X, n)$. The induced action on the cohomology algebra (with rational coefficients) $H^{*}(F(X, n))$ has as the invariant part the rational cohomology of $C(X, n)$.

For $X$ a smooth complex projective variety, I. Križ $\mathrm{K}$ constructed a rational model $E(X, n)$ for $F(X, n)$, a simplified version of the Fulton-MacPherson model FM. Let us remind the construction of Križ. We denote by $p_{i}^{*}: H^{*}(X) \rightarrow H^{*}\left(X^{n}\right)$ and $p_{i j}^{*}: H^{*}\left(X^{2}\right) \rightarrow H^{*}\left(X^{n}\right)$ (for $i \neq j$ ) the pullbacks of the obvious projections and by $m$ the complex dimension of $X$. The model $E(X, n)$ is defined as follows: as an algebra, $E(X, n)$ is isomorphic to the exterior algebra with generators $G_{i j}, 1 \leq$ $i, j \leq n$ (of degree $\left|G_{i j}\right|=2 m-1$ ) and coefficients in $H^{*}(X)^{\otimes n}$ modulo the relations

$$
\begin{aligned}
& G_{j i} \quad=G_{i j}, \\
& p_{j}^{*}(x) G_{i j}=p_{i}^{*}(x) G_{i j}, \quad(i<j), x \in H^{*}(X), \\
& G_{i k} G_{j k}=G_{i j} G_{j k}-G_{i j} G_{i k}, \quad(i<j<k) .
\end{aligned}
$$

The differential $d$ is given by $\left.d\right|_{H^{*}(X) \otimes n}=0$ and $d\left(G_{i j}\right)=p_{i j}^{*}(\Delta)$, where $\Delta=$ $w \otimes 1+\ldots+1 \otimes w \in H^{*}(X) \otimes H^{*}(X)$ denotes the class of the diagonal and $w \in H^{2 m}(X)$ is the fundamental class. This model is a differential bigraded algebra $E(X, n)=\bigoplus_{k, q} E_{q}^{k}(X, n)$ : the lower degree $q$ (called the exterior degree) is given by the number of exterior generators $G_{i j}$, and the upper degree $k$ is given by the total degree. The multiplication is homogeneous and the differential has bidegree $\left(\begin{array}{l}+1 \\ -1\end{array}\right)$

$$
E_{q}^{k} \otimes E_{q^{\prime}}^{k^{\prime}} \rightarrow E_{q+q^{\prime}}^{k+k^{\prime}}, d: E_{q}^{k} \rightarrow E_{q-1}^{k+1},
$$

Key words and phrases. Configurations in complex projective spaces, Križ model, representations of symmetric group.

2010 AMS Classification Primary: 55R80, 20C30; Secondary: 55P62, 13 A50.

This research is partially supported by Higher Education Commission, Pakistan. 
therefore the cohomology algebra of the configuration space, $H_{*}^{*}=H_{*}^{*}(F(X, n))$, is a bigraded algebra and we will compute the two variable Poincaré polynomial

$$
P_{F(X, n)}(t, s)=\sum_{k, q \geq 0}\left(\operatorname{dim} H_{q}^{k}\right) t^{k} s^{q}
$$

The canonical basis for the Križ model is given by products of exterior elements $G_{I_{*} J_{*}}=G_{i_{1} j_{1}} G_{i_{2} j_{2}} \ldots G_{i_{q} j_{q}}$, where $I_{*}=\left(i_{1}, \ldots, i_{q}\right), J_{*}=\left(j_{1}, \ldots, j_{q}\right)$, satisfying $i_{a}<j_{a}(a=1,2, \ldots, q)$ and $j_{1}<j_{2}<\ldots<j_{q}$, with scalars $x_{*}=x_{1} \otimes x_{2} \otimes \ldots \otimes x_{n}$ (the factors belong to a fixed basis of $H^{*}(X)$ ), and $x_{j}=1$ if $j \in J_{*}$, see [B], BMP] or $\mathrm{AAB}$ for more details).

The symmetric group $\mathcal{S}_{n}$ acts on each bigraded component $E_{q}^{k}(X, n)$ by permuting the factors in $H^{*}\left(X^{n}\right)=H^{* \otimes n}$ and changing the indices of the exterior generators (see $[\mathrm{FM}]$ and $[\mathrm{K}]$ ): for an arbitrary permutation $\sigma \in \mathcal{S}_{n}$

$$
\sigma\left(p_{1}^{*}\left(x_{1}\right) \ldots p_{n}^{*}\left(x_{n}\right) G_{I_{*} J_{*}}\right)=p_{\sigma(1)}^{*}\left(x_{1}\right) \ldots p_{\sigma(n)}^{*}\left(x_{n}\right) G_{\sigma\left(I_{*}\right) \sigma\left(J_{*}\right)} .
$$

The differential is equivariant (see [AAB]), and therefore we can split the differential algebra $\left(E_{*}^{*}, d\right)$ into isotypical parts corresponding to partitions $\lambda$ of $n, \lambda \vdash n$,

$$
\left(E_{*}^{*}(X, n), d\right) \cong \bigoplus_{\lambda \vdash n}\left(E_{*}^{*}(X, n)(\lambda), d_{\lambda}\right) .
$$

In FTh Felix and Thomas proved that for even dimensional closed manifold $M$ the rational Betti numbers of $C(M, n)$ are determined by the graded cohomology algebra $H^{*}(M ; \mathbb{Q})$, and, as an application, they computed the Betti numbers of $C\left(\mathbb{C} P^{3}, 3\right)$. A presentation of the rational cohomology of $C\left(\mathbb{C} P^{2}, n\right)$ appears in [FT] by analyzing the $\mathcal{S}_{n}$-invariant part of the spectral sequence converging to $H^{*}(F(M, n))$, introduced by Cohen and Taylor [CT]; see also [FOT]. In [S] Križ model and some natural fibrations are used to compute $H^{*}\left(F\left(\mathbb{C} P^{m}, 2\right)\right)$ for an arbitrary finite $m$ and $H^{*}\left(F\left(\mathbb{C} P^{m}, 3\right)\right)$ for $m \leq 4$. In [FZ] Feichtner and Ziegler described the cohomology algebra of $F\left(\mathbb{C} P^{1}, n\right)$ with integer coefficients. The symmetric structure of $H^{*}\left(F\left(\mathbb{C} P^{1}, n\right)\right)$ was considered in $\mathrm{AAB}$.

In this paper we analyze the cohomology of various configuration spaces of three points in complex projective spaces $\mathbb{C} P^{m}$ for $m \geq 2$, including the infinite dimensional space, using the representation theory of the Križ model and some associated spectral sequences.

In Section 2 we describe the symmetric structure of the Križ model $E_{*}^{*}=$ $E_{*}^{*}\left(\mathbb{C} P^{m}, 3\right)$. The irreducible modules corresponding to the partition $\lambda$ will be denoted by $V(\lambda)$, as in $\left[\mathrm{FH}\right.$. The multiplicities of the irreducible $\mathcal{S}_{3}$-modules in $E_{q}^{k}$ are given in terms of partitions and bounded partitions of an integer into three parts, see Proposition 2.1. Explicit formulae for these numbers are given in the Appendix. In the stable cases, $m \geq 5$, the pairs $(k, q)$ with non-zero $E_{q}^{k}(\lambda)$ and those with non-zero $H_{q}^{k}(\lambda)$ are given in the next diagrams (the former pairs are marked with small circles and the latter with bullets; the dots mark the places where $E_{q}^{k} \neq 0$ and $\left.E_{q}^{k}(\lambda)=0\right)$. 

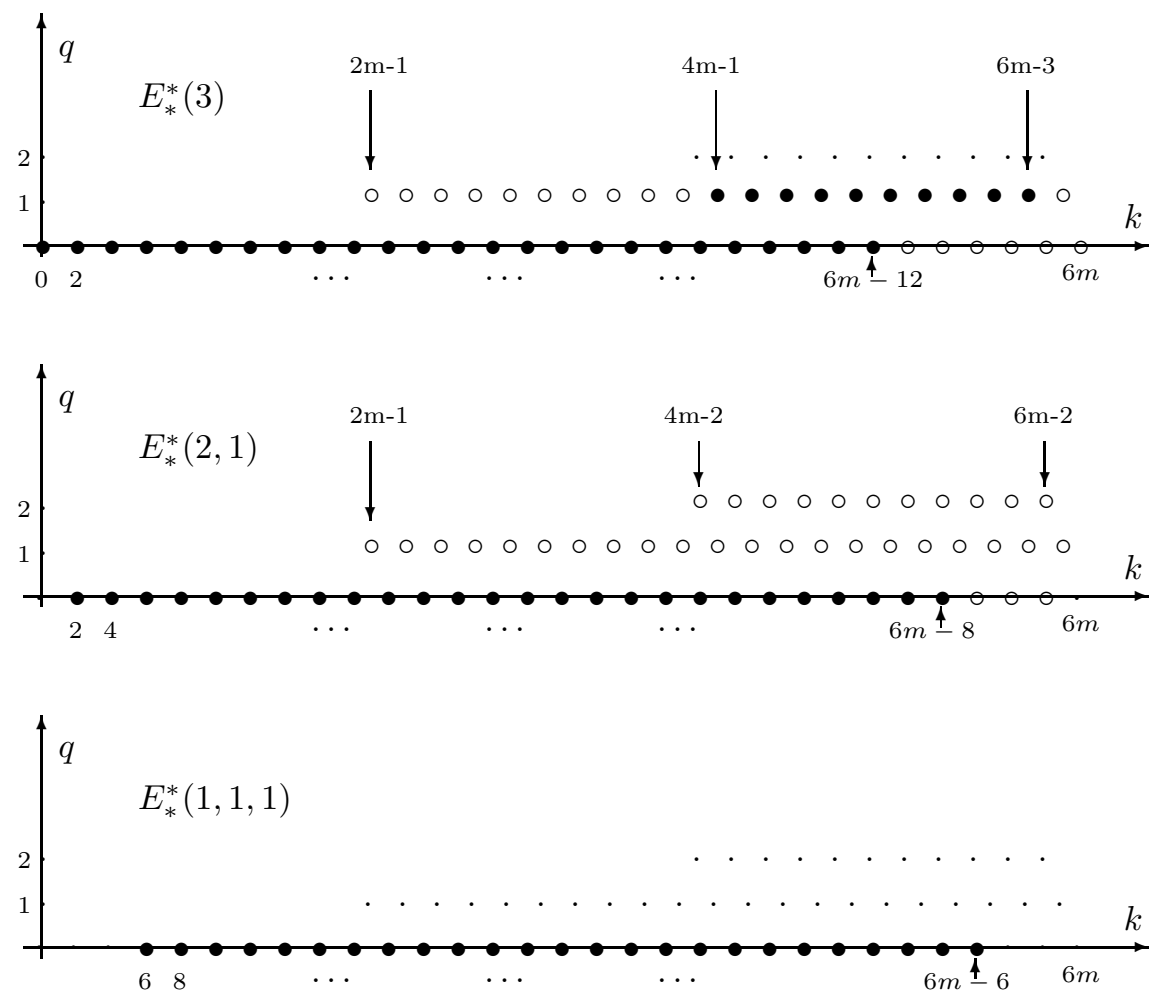

In Section 3 we compute the Betti numbers of each isotypical part of $E_{*}^{*}\left(\mathbb{C} P^{m}, 3\right)$. Summing up their Poincaré polynomials we obtain

Theorem 1.1. The two variable Poincaré polynomial for the ordered configuration space $F\left(\mathbb{C} P^{m}, 3\right)$ is given by:

$$
P_{F\left(\mathbb{C} P^{m}, 3\right)}(t, s)=C_{m-1}^{3}(t)+s t^{4 m-1} C_{m-1}(t) .
$$

In this theorem and the next ones we use the notation

$$
r_{m}(x, y)=x^{m}+x^{m-1} y+\ldots+y^{m}, C_{m}(t)=r_{m}\left(1, t^{2}\right)=1+t^{2}+\ldots+t^{2 m} .
$$

In the Appendix one can find the proof of following:

Corollary 1.2. The sequences of even and odd Betti numbers of the configuration space $F\left(\mathbb{C} P^{m}, 3\right)$ and also the sequences of its isotypic parts are unimodal:

$$
\begin{aligned}
& \beta_{0} \leq \beta_{2} \leq \ldots \leq \beta_{2 i} \geq \beta_{2 i+2} \geq \ldots \quad \ldots \quad \beta_{6 m-2} \geq \beta_{6 m}, \\
& \beta_{1} \leq \beta_{3} \leq \ldots \leq \beta_{2 j-1} \geq \beta_{2 j+1} \geq \ldots \quad \geq \beta_{6 m-3} \geq \beta_{6 m-1} .
\end{aligned}
$$

In Section 4 the multiplicative structure of the cohomology algebras $H^{*}\left(F\left(\mathbb{C} P^{m}, 3\right)\right)$ and $H^{*}\left(C\left(\mathbb{C} P^{m}, 3\right)\right)$ is analyzed.

Theorem 1.3. The cohomology algebra $H^{*}\left(F\left(\mathbb{C} P^{m}, 3\right)\right)$ has the presentation (as a graded commutative algebra):

$$
\left\langle\alpha_{1}, \alpha_{2}, \alpha_{3}, \eta \mid \alpha_{1}^{m+1}, r_{m}\left(\alpha_{i}, \alpha_{j}\right),\left(\alpha_{i}-\alpha_{j}\right) \eta, \alpha_{1}^{m} \eta\right\rangle
$$

where $\left|\alpha_{i}\right|=2,|\eta|=4 m-1$. 
From the Newton's version of the fundamental theorem of symmetric polynomials, there are uniquely defined polynomials $P_{k} \in \mathbb{Q}\left[X_{1}, X_{2}, X_{3}\right]$ such that

$$
X_{1}^{k}+X_{2}^{k}+X_{3}^{k}=P_{k}\left(X_{1}+X_{2}+X_{3}, X_{1}^{2}+X_{2}^{2}+X_{3}^{2}, X_{1}^{3}+X_{2}^{3}+X_{3}^{3}\right),
$$

and these are used in Theorem 1.4 and in Section 4.

Theorem 1.4. The cohomology algebra $H^{*}\left(C\left(\mathbb{C} P^{m}, 3\right)\right)$ has the presentation (as a graded commutative algebra):

$$
\left\langle\begin{array}{c|c}
\tau_{1}, \tau_{2}, \tau_{3}, & \begin{array}{c}
P_{m+1}, P_{m+2}, P_{m+3},\left(\tau_{1}^{2}-3 \tau_{2}\right) \eta,\left(\tau_{1} \tau_{2}-3 \tau_{3}\right) \eta, P_{m} \eta \\
\eta
\end{array} \\
(m+1) P_{m}-\sum_{i=0}^{m} P_{i} P_{m-i}, \sum_{i=1}^{m-1} P_{i} P_{m-i}, \sum_{i=2}^{m-2} P_{i} P_{m-i}
\end{array}\right\rangle
$$

where $\left|\tau_{i}\right|=2 i(i=1,2,3),|\eta|=4 m-1$ and $P_{k}=P_{k}\left(\tau_{1}, \tau_{2}, \tau_{3}\right)$.

In the next Section we describe the "stable cohomology" of the configuration spaces: as the $\operatorname{limit}_{\lim _{m}} F\left(\mathbb{C} P^{m}, n\right)=F\left(\mathbb{C} P^{\infty}, n\right)$ has the homotopy type of $\left(\mathbb{C} P^{\infty}\right)^{n}$, see Theorem 5.1, we obtain

Theorem 1.5. The cohomology algebras of the ordered and unordered configuration spaces of the infinite complex projective space are given by

$$
\begin{aligned}
& H^{*}\left(F\left(\mathbb{C} P^{\infty}, n\right)\right) \cong \mathbb{Q}\left[x_{1}, x_{2}, \ldots, x_{n}\right], \\
& H^{*}\left(C\left(\mathbb{C} P^{\infty}, n\right)\right) \cong \mathbb{Q}\left[\sigma_{1}, \sigma_{2}, \ldots, \sigma_{n}\right],
\end{aligned}
$$

where $\left|x_{i}\right|=2$ and $\left|\sigma_{i}\right|=2 i$.

and the corollary

Corollary 1.6. The inclusion $\mathbb{C} P^{m} \hookrightarrow \mathbb{C} P^{\infty}$ induces an isomorphism

$$
H^{k}\left(F\left(\mathbb{C} P^{\infty}, n\right)\right) \cong H^{k}\left(F\left(\mathbb{C} P^{m}, n\right)\right)
$$

for $0 \leq k \leq 2 m-1$.

In Section 6 we compute the Betti numbers of the spaces of configurations of three collinear points and configurations of three non-collinear points (see $[\mathrm{BP}]$ for the fundamental groups of these spaces):

$$
\begin{aligned}
& F_{c}\left(\mathbb{C} P^{m}, 3\right)=\left\{(p, q, r) \in F\left(\mathbb{C} P^{m}, 3\right) \mid p, q, r \text { are collinear }\right\}, \\
& F_{n c}\left(\mathbb{C} P^{m}, 3\right)=F\left(\mathbb{C} P^{m}, 3\right) \backslash F_{c}\left(\mathbb{C} P^{m}, 3\right),
\end{aligned}
$$

Theorem 1.7. The Poincaré series of the space of configurations of three collinear points in $\mathbb{C} P^{m}$ is given by:

$$
P_{F_{c}\left(\mathbb{C} P^{m}, 3\right)}(t)=\left(1+t^{2 m+1}\right) C_{m-1}(t)=\frac{\left(1+t^{2 m+1}\right)\left(1-t^{2 m}\right)}{1-t^{2}},
$$

in the finite case. If $m$ is infinite we have

$$
P_{F_{c}\left(\mathbb{C} P^{\infty}, 3\right)}(t)=\frac{1}{1-t^{2}} .
$$

Theorem 1.8. The Poincaré series of the ordered configuration space of three non-collinear points in complex projective space is given as:

$$
P_{F_{n c}\left(\mathbb{C} P^{m}, 3\right)}(t)=C_{m-2}(t) \cdot C_{m-1}(t) \cdot C_{m}(t)=\frac{\left(1-t^{2 m-2}\right)\left(1-t^{2 m}\right)\left(1-t^{2 m+2}\right)}{\left(1-t^{2}\right)^{3}},
$$

in the finite case. If $m$ is infinite we have

$$
P_{F_{n c}\left(\mathbb{C} P^{\infty}, 3\right)}(t)=\frac{1}{\left(1-t^{2}\right)^{3}} .
$$




\section{The $\mathcal{S}_{3}$-Decomposition of $E_{*}^{*}\left(\mathbb{C} P^{m}, 3\right)$}

The equation $a+b+c=k(a, b, c$ are non-negative integers) has three types of solutions: the first type when $a, b, c$ are pairwise distinct, the second type when the set $\{a, b, c\}$ has two elements, and the third type when $a=b=c$ (if $3 \mid k$ ). The symmetric group $\mathcal{S}_{3}$ acts on the set of triplets $(a, b, c)$ and linearizing this action we find the next $\mathcal{S}_{3}$-modules corresponding to the three types:

$$
\begin{aligned}
& \mathbb{Q}\left[\mathcal{S}_{3}\right]\langle a, b, c\rangle \cong V(3) \oplus 2 V(2,1) \oplus V(1,1,1), \\
& \mathbb{Q}\left[\mathcal{S}_{3}\right]\langle a, a, b\rangle \cong V(3) \oplus V(2,1), \\
& \mathbb{Q}\left[\mathcal{S}_{3}\right]\langle a, a, a\rangle \cong V(3) .
\end{aligned}
$$

We denote by $P_{3}(k)$ the cardinality of partitions of $k$ into three non-negative parts

$$
P_{3}(k)=\operatorname{card}\{(a, b, c) \mid a \geq b \geq c \geq 0, a+b+c=k\}
$$

and also by $P_{3, \leq m}(k)$ the cardinality of bounded partitions

$$
P_{3, \leq m}(k)=\operatorname{card}\{(a, b, c) \mid m \geq a \geq b \geq c \geq 0, a+b+c=k\} .
$$

See the Appendix for the computation of these numbers and some of their elementary properties.

In the next statement we will use the notation $r_{3}(k)=1$ if $3 \mid k$ and 0 otherwise; the component $E_{q}^{k}\left(\mathbb{C} P^{m}, 3\right)$ is denoted shortly $E_{q}^{k}$. From now on, $H^{*}$ will denote the cohomology algebra $H^{*}\left(\mathbb{C} P^{m} ; \mathbb{Q}\right) \cong \mathbb{Q}[x] /\left\langle x^{m+1}\right\rangle$, where $|x|=2$; the canonical basis is $\left\{1, x, \ldots, x^{m}\right\}$ and $\Delta=x^{m} \otimes 1+x^{m-1} \otimes x+\ldots+1 \otimes x^{m}$.

Proposition 2.1. The non-zero bigraded components $E_{q}^{k}$ are decomposed into irreducible $\mathcal{S}_{3}$-modules with multiplicities from the following table:

\begin{tabular}{|cll|c|c|c|}
\hline & & $V(3)$ & $V(2,1)$ & $V(1,1,1)$ \\
\hline$E_{2}^{2(2 m-1)+2 k} \cong E_{2}^{2(3 m-1)-2 k}$ & $(0 \leq 2 k \leq m)$ & - & 1 & - \\
$E_{1}^{(2 m-1)+2 k} \cong E_{1}^{(6 m-1)-2 k}$ & $(0 \leq k \leq m)$ & $k+1$ & $k+1$ & - \\
$E_{0}^{2 k} \cong E_{0}^{6 m-2 k}$ & $(0 \leq 2 k \leq 3 m)$ & $\mu_{3}(k)$ & $\mu_{2,1}(k)$ & $\mu_{1,1,1}(k)$ \\
\hline
\end{tabular}

where

$$
\begin{aligned}
\mu_{3}(k) & =P_{3, \leq m}(k) \\
\mu_{2,1}(k) & =2 P_{3, \leq m}(k)-\left(\left\lfloor\frac{k}{2}\right\rfloor+1+r_{3}(k)-\max \left\{0,\left\lceil\frac{k-m}{2}\right\rceil\right\}\right) \\
& =P_{3, \leq m}(k)+P_{3, \leq m-2}(k-3)-r_{3}(k), \\
\mu_{1,1,1}(k) & =P_{3, \leq m}(k)-\left(\left\lfloor\frac{k}{2}\right\rfloor+1-\max \left\{0,\left\lceil\frac{k-m}{2}\right\rceil\right\}\right) \\
& =P_{3, \leq m-2}(k-3) .
\end{aligned}
$$

Proof. All the cohomology classes in $H^{*}$ are of even degree, hence $E_{q}^{k}=0$ if $k+q \equiv 1$ (mod 2). For large values of $k$ we use the $\mathcal{S}_{3}$-equivariant Poincaré duality, see [AAB].

0) The action of $\mathcal{S}_{3}$ on $E_{0}^{2 k}$ is equivalent with the action on the set of non negative solutions of the equation $a+b+c=k, a, b, c \leq m$ and the decompositions of the three types were given above. The multiplicity $\mu_{1,1,1}(k)$ is given by the number of solutions of the equation

$$
a+b+c=k, \quad m \geq a>b>c \geq 0,
$$

and this number equals the number $P_{3, \leq m}(k)$ minus the number of solutions of the equation

$$
2 a+b=k, \quad m \geq a, b \geq 0
$$


and this gives the interval $\max \left\{0,\left\lceil\frac{k-m}{2}\right\rceil\right\} \leq a \leq\left\lfloor\frac{k}{2}\right\rfloor$, (here $k \leq \frac{3 m}{2}$ ). The second formula is a consequence of the bijection

$$
\left\{\begin{aligned}
(a, b, c) \mid \begin{array}{l}
a+b+c=k, \\
m \geq a>b>c \geq 0
\end{array} \\
(a, b, c)
\end{aligned} \quad \Leftrightarrow\left\{\begin{array}{c}
(\alpha, \beta, \gamma) \mid \begin{array}{l}
\alpha+\beta+\gamma=k-3, \\
m-2 \geq \alpha \geq \beta \geq \gamma \geq 0 \\
(a-2, b-1, c) .
\end{array}
\end{array}\right\}\right.
$$

The multiplicity $\mu_{2,1}(k)$ is given by the total number of solutions of the first two types, $P_{3, \leq m}(k)-r_{3}(k)$, plus the number of solutions of the first type, $\mu_{1,1,1}(k)$.

1) The elements of the canonical basis $E_{1}^{(2 m-1)+2 k}$ correspond to the marked graphs $\left(\Gamma_{(2,1)},\left(x^{h^{\prime}}, x^{h^{\prime \prime}}\right)\right)$ such that $h^{\prime}+h^{\prime \prime}=k$, where $0 \leq k \leq m$ (see [AAB for a marked version of the graphs introduced in [LS]). The possible marks $\left(h^{\prime}, h^{\prime \prime}\right)$ are $(0, k),(1, k-1), \ldots,(k, 0)$ and the corresponding marked graphs are:

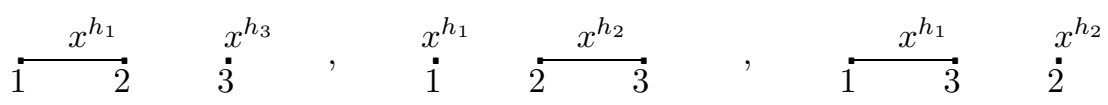

The transposition (12) preserves the first marked graph and the 3-cycles have no contribution to the trace, hence the character of this representation gives the decomposition $V(3) \oplus V(2,1)$.

2 ) For $E_{2}^{2(2 m-1)+2 k}$ we have a unique type of associated marked graphs, $\left(\Gamma_{(3)}, x^{h_{1}}\right)$, giving the irreducible module $V(2,1)$ :

$$
\left(\Gamma_{(3)}, x^{h_{1}}\right): \quad \stackrel{x^{h_{1}}}{\stackrel{2}{2} \quad, \quad \frac{x^{h_{1}}}{2}}
$$

\section{Computing the Differentials}

For three points in $\mathbb{C} P^{m}$ we have the decomposition

$$
\left(E_{*}^{*}, d\right)=\left(E_{*}^{*}(3), d_{3}\right) \oplus\left(E_{*}^{*}(2,1), d_{2,1}\right) \oplus\left(E_{*}^{*}(1,1,1), d_{1,1,1}\right)
$$

corresponding to the three partitions of 3 . We analyze the properties of the differential in each of these three components.

Lemma 3.1. For $0 \leq k \leq m-1$ the differential $d_{3}$ is injective

$$
d: E_{1}^{(2 m-1)+2 k}(3) \longmapsto E_{0}^{2 m+2 k}(3) .
$$

Proof. We introduce filtrations on both $E_{1}^{(2 m-1)+2 k}(3)$ and $E_{0}^{2 m+2 k}(3)$. For a triple $\alpha=(a, b, c)$ with $|\alpha|=a+b+c=m+k$ and $m \geq a \geq b \geq c \geq 0$ we denote by $v_{\alpha}$ the element of $E_{0}^{2 m+2 k}$

$$
v_{\alpha}=\sum_{\pi \in \mathcal{S}_{3}} \pi\left(x^{a} \otimes x^{b} \otimes x^{c}\right) .
$$

It is obvious that $\left\{v_{\alpha}|| \alpha \mid=m+k\right\}$ is a basis of $E_{0}^{2 m+2 k}(3)$. Using the lexicographic order on $\alpha$ 's we define an increasing filtration on $E_{0}^{2 m+2 k}(3)$ by

$$
\mathcal{T}_{\alpha}=\bigoplus_{\beta \leq \alpha} \mathbb{Q}\left\langle v_{\beta}\right\rangle
$$


We choose the basis of the module $E_{1}^{(2 m-1)+2 k}(3)$ given by:

$$
\begin{aligned}
\sigma_{a}= & \left(x^{a} \otimes 1 \otimes x^{k-a}+x^{k-a} \otimes 1 \otimes x^{a}\right) G_{12}+\left(x^{a} \otimes x^{k-a} \otimes 1+x^{k-a} \otimes x^{a} \otimes 1\right) G_{13} \\
& +\left(x^{k-a} \otimes x^{a} \otimes 1+x^{a} \otimes x^{k-a} \otimes 1\right) G_{23} \\
\delta_{a}= & \left(x^{a} \otimes 1 \otimes x^{k-a}-x^{k-a} \otimes 1 \otimes x^{a}\right) G_{12}+\left(x^{a} \otimes x^{k-a} \otimes 1-x^{k-a} \otimes x^{a} \otimes 1\right) G_{13} \\
& +\left(x^{k-a} \otimes x^{a} \otimes 1-x^{a} \otimes x^{k-a} \otimes 1\right) G_{23},
\end{aligned}
$$

where $\left\lceil\frac{k}{2}\right\rceil \leq a \leq k$.

We define an increasing filtration on $E_{1}^{(2 m-1)+2 k}(3)$ in two steps:

$$
\mathcal{D}_{a}=\bigoplus_{b \leq a} \mathbb{Q}\left\langle\delta_{b}\right\rangle \text { and } \mathcal{G}_{a}=\mathcal{D}_{k} \bigoplus\left(\underset{b \leq a}{\oplus} \mathbb{Q}\left\langle\sigma_{b}\right\rangle\right)
$$

The leading monomial of $d\left(\delta_{a}\right)$ is given by the projection of $d\left(\delta_{a}\right)$ on the monomials containing $x^{m}$ or $x^{m-1}$ on the first position:

$$
\begin{aligned}
\operatorname{pr}_{(m, m-1)}\left(d\left(\delta_{a}\right)\right)= & x^{m} \otimes x^{a} \otimes x^{k-a}+x^{m-1} \otimes x^{a+1} \otimes x^{k-a}- \\
& -x^{m} \otimes x^{k-a} \otimes x^{a}-x^{m-1} \otimes x^{k-a+1} \otimes x^{a}+ \\
& +x^{m} \otimes x^{k-a} \otimes x^{a}+x^{m-1} \otimes x^{k-a} \otimes x^{a+1}- \\
& -x^{m} \otimes x^{a} \otimes x^{k-a}-x^{m-1} \otimes x^{a} \otimes x^{k-a+1}= \\
= & x^{m-1} \otimes x^{a+1} \otimes x^{k-a}+x^{m-1} \otimes x^{k-a} \otimes x^{a+1}- \\
& -x^{m-1} \otimes x^{k-a+1} \otimes x^{a}-x^{m-1} \otimes x^{a} \otimes x^{k-a+1}
\end{aligned}
$$

On the other hand,

$$
\begin{aligned}
\operatorname{pr}_{(m, m-1)}\left(d\left(\sigma_{a}\right)\right)= & x^{m} \otimes x^{a} \otimes x^{k-a}+x^{m-1} \otimes x^{a+1} \otimes x^{k-a}+ \\
& +x^{m} \otimes x^{k-a} \otimes x^{a}+x^{m-1} \otimes x^{k-a+1} \otimes x^{a}+ \\
& +x^{m} \otimes x^{k-a} \otimes x^{a}+x^{m-1} \otimes x^{k-a} \otimes x^{a+1}+ \\
& +x^{m} \otimes x^{a} \otimes x^{k-a}+x^{m-1} \otimes x^{a} \otimes x^{k-a+1}
\end{aligned}
$$

hence $d\left(\sigma_{a}\right)$ has $x^{m} \otimes x^{a} \otimes x^{k-a}$ as the leading monomial. These computations imply the injectivity of the map induced by the differential $d$ between the graded modules associated to the filtrations $\left(\mathcal{D}_{*}, \mathcal{G}_{*}\right)$ and $\mathcal{T}_{*}$ :

$$
\left(\underset{a=\left\lceil\frac{k}{2}\right\rceil}{\stackrel{k}{\oplus}} \mathcal{D}_{a} / \mathcal{D}_{a-1}\right) \bigoplus\left(\underset{a=\left\lceil\frac{k}{2}\right\rceil}{\stackrel{k}{\oplus}} \mathcal{G}_{a} / \mathcal{G}_{a-1}\right) \stackrel{d}{\longmapsto} \bigoplus_{\alpha} \mathcal{T}_{\alpha} / \mathcal{T}_{<\alpha}
$$

(here $\mathcal{D}_{\left\lceil\frac{k}{2}\right\rceil-1}=0$ and $\mathcal{G}_{\left\lceil\frac{k}{2}\right\rceil-1}=\mathcal{D}_{k}$.) Therefore the map $d: E_{1}^{(2 m-1)+2 k}(3) \rightarrow$ $E_{0}^{2 m+2 k}(3)$ is injective for $k \leq m-1$.

Lemma 3.2. For $m \leq k \leq 2 m-1$, the dimension of the space of cocycles in $E_{1}^{(2 m-1)+2 k}(3)$ is at most one.

Proof. We consider the basis of the module $E_{1}^{(2 m-1)+2 k}(3)$ given by the vectors $\sigma_{a}$ and $\delta_{a}$, where $\left\lceil\frac{k}{2}\right\rceil \leq a \leq m$ and the same filtration as defined in the previous lemma. We prove that the induced map on the hyperplane $\mathcal{G}_{m-1}$ is injective. Similar computations for the leading terms in the differentials of the elements of the basis $\left\{\sigma_{*}, \delta_{*}\right\}$ give that the differential of $\delta_{a}$, for $a \neq m$, has the highest term $x^{m-1} \otimes x^{a+1} \otimes x^{k-a}$ (for $\delta_{m}$ the highest term is $x^{m} \otimes x^{m} \otimes x^{k-m}$ ). On the other hand, the leading monomial in the differential of $\sigma_{a}$ is $x^{m} \otimes x^{a} \otimes x^{k-a}$. Thus the leading monomials do not interfere except the ones corresponding to $\delta_{m}$ and $\sigma_{m}$ and the differential is injective on the hyperplane

$$
\mathcal{G}_{m-1}=\mathcal{D}_{m} \bigoplus\left(\underset{b \leq m-1}{\oplus} \mathbb{Q}\left\langle\sigma_{b}\right\rangle\right)
$$


Remark 3.3. For $k=2 m, d: E_{1}^{6 m-1}(3) \rightarrow E_{0}^{6 m}(3)$ is injective since both the spaces are one dimensional and the differential is not zero. In this case the unique generator is $\sigma_{m}=2\left(x^{m} \otimes 1 \otimes x^{m} G_{12}+x^{m} \otimes x^{m} \otimes 1 G_{13}+x^{m} \otimes x^{m} \otimes 1 G_{23}\right) \in E_{1}^{6 m-1}(3)$. More generally, the right side of the trapezoid $E_{*}^{*}$ is acyclic, see [AAB].

The coordinates of the vector $W$ in the next lemma correspond to the frame $x^{m} \otimes 1 \otimes 1 G_{12}+x^{m} \otimes 1 \otimes 1 G_{13}+1 \otimes x^{m} \otimes 1 G_{23}, x^{m-1} \otimes 1 \otimes x G_{12}+x^{m-1} \otimes x \otimes$ $1 G_{13}+x \otimes x^{m-1} \otimes 1 G_{23}, \ldots, 1 \otimes 1 \otimes x^{m} G_{12}+1 \otimes x^{m} \otimes 1 G_{13}+x^{m} \otimes 1 \otimes 1 G_{23}$ of $E_{1}^{4 m-1}(3)$.

Lemma 3.4. The kernel of $d: E_{1}^{4 m-1}(3) \rightarrow E_{0}^{4 m}(3)$ is one dimensional and is generated by the vector $W=(2 m, 2 m-3,2 m-6, \ldots,-m+3,-m)$.

Proof. From the previous lemma the dimension of the kernel is $\leq 1$, and now we compute the differential of $W$ :

$$
\begin{aligned}
W= & 2 m\left(x^{m} \otimes 1 \otimes 1 G_{12}+x^{m} \otimes 1 \otimes 1 G_{13}+1 \otimes x^{m} \otimes 1 G_{23}\right)+ \\
& +(2 m-3)\left(x^{m-1} \otimes 1 \otimes x G_{12}+x^{m-1} \otimes x \otimes 1 G_{13}+x \otimes x^{m-1} \otimes 1 G_{23}\right)+\ldots \\
& +(2 m-3 a)\left(x^{m-a} \otimes 1 \otimes x^{a} G_{12}+x^{m-a} \otimes x^{a} \otimes 1 G_{13}+x^{a} \otimes x^{m-a} \otimes 1 G_{23}\right) \\
& +\ldots+(2 m-3 m)\left(1 \otimes 1 \otimes x^{m} G_{12}+1 \otimes x^{m} \otimes 1 G_{13}+x^{m} \otimes 1 \otimes 1 G_{23}\right) .
\end{aligned}
$$

A monomial $x^{a} \otimes x^{b} \otimes x^{c}, m \geq a \geq b \geq c \geq 0, a+b+c=2 m$ appears at most once in the differential of each of the rows of $W$, and the total sum of its coefficients equals

$$
(2 m-3 a)+(2 m-3 b)+(2 m-3 c)=6 m-3(a+b+c)=0 .
$$

Hence $d W=0$ and the kernel of $d: E_{1}^{4 m-1}(3) \rightarrow E_{0}^{4 m}(3)$ is one dimensional.

Lemma 3.5. For $m \leq k \leq 2 m-1$, the space of cocycles in $E_{1}^{(2 m-1)+2 k}(3)$ is one dimensional.

Proof. Multiplying the cocycle $W \in E_{1}^{(2 m-1)+2 m}(3)$ by the symmetric scalar $t_{k}=$ $x^{k} \otimes 1 \otimes 1+1 \otimes x^{k} \otimes 1+1 \otimes 1 \otimes x^{k}, k=0, \ldots, m-1$, we obtain an $\mathcal{S}_{3}$ invariant cocycle which is not zero, since the coefficient of $x^{m} \otimes 1 \otimes x^{k} G_{12}$ in $t_{k} W$ is $2 m+2(2 m-3 k)=$ $6 m-6 k$, which is non-zero for $k \neq m$.

Remark 3.6. The element $t_{m} W$ is also a cocycle, but $t_{m} W=0$.

Now we can compute the Poincaré polynomial for the unordered configuration space $C\left(\mathbb{C} P^{m}, 3\right)$ :

Proposition 3.7. The Poincaré polynomial of the unordered configuration space $C\left(\mathbb{C} P^{m}, 3\right)$ is given by:

$$
\begin{aligned}
P_{C\left(\mathbb{C} P^{m}, 3\right)}(t, s)= & \sum_{k=0}^{m-1}\left(P_{3, \leq m}(k)\right) t^{2 k}+\sum_{k=m}^{2 m-1}\left(P_{3, \leq m}(k)+m-k-1\right) t^{2 k}+ \\
& +\sum_{k=2 m}^{3 m-1}\left(P_{3, \leq m}(k)-3 m+k\right) t^{2 k}+\sum_{k=2 m}^{3 m-1} s t^{2 k-1}= \\
= & 1+t^{2}+2 t^{4}+3 t^{6}+4 t^{8}+5 t^{10}+7 t^{12}+8 t^{14}+10 t^{16}+ \\
& +12 t^{18}+\ldots+2 t^{6 m-16}+t^{6 m-14}+t^{6 m-12}+ \\
& +s\left(t^{4 m-1}+t^{4 m+1}+\ldots t^{6 m-5}+t^{6 m-3}\right) .
\end{aligned}
$$

where, in the second expansion, the coefficient of $t^{6 m-12}$ is stable for $m \geq 5$ (the coefficient of $t^{6 m-14}$ is stable for $m \geq 6$ and the first terms for $m \geq 10$ ). 
Proof. The proof follows from the computations of the multiplicity of the trivial representation $V(3)$ in $E_{1}^{(2 m-1)+2 k}$ and $E_{0}^{2 k}$ in Section 2 and the Lemmas 3.1 and 3.5. For $k=3 m-l, 0 \leq l \leq 6$, we have $P_{3, \leq m}(k)=P_{3, \leq m}(l)$ so

$$
P_{3, \leq m}(k)-3 m+k=P_{3, \leq m}(l)-l
$$

which is zero for $0 \leq l \leq 5$; for $l=6$ and $m \geq 6$ we have $P_{3, \leq m}(6)=7$, giving $\beta_{6 m-12}=1$. For $m=5$ the monomial $t^{18}$ belongs to the second sum and its coefficient is 1 . The initial terms in the second expansion are stable for $m \geq 10$ : $P_{3, \leq m}(10)=P_{3}(10)$.

Remark 3.8. For $m=2,3,4$ we have

$$
\begin{aligned}
& P_{C\left(\mathbb{C} P^{2}, 3\right)}(t, s)=1+t^{2}+t^{4}+s\left(t^{7}+t^{9}\right) \\
& P_{C\left(\mathbb{C} P^{3}, 3\right)}(t, s)=1+t^{2}+2 t^{4}+2 t^{6}+t^{8}+s\left(t^{11}+t^{13}+t^{15}\right), \\
& P_{C\left(\mathbb{C} P^{4}, 3\right)}(t, s)=1+t^{2}+2 t^{4}+3 t^{6}+3 t^{8}+2 t^{10}+2 t^{12}+s\left(t^{15}+t^{17}+t^{19}+t^{21}\right) .
\end{aligned}
$$

The differentials of the $(2,1)$-component have similar properties.

Lemma 3.9. For $0 \leq k \leq m-1$, the differential $d_{2,1}$ is injective

$$
d: E_{1}^{(2 m-1)+2 k}(2,1) \longmapsto E_{0}^{2 m+2 k}(2,1) .
$$

Proof. The space $E_{1}^{(2 m-1)+2 k}(2,1)$ consists of copies of $V(2,1), \mathbf{V}_{a}$ (where $a$ runs from 0 to $k$ ), generated by vectors of the form:

$$
\mathbf{V}_{a}:\left\{\begin{array}{l}
v_{1, a}=x^{k-a} \otimes 1 \otimes x^{a} G_{12}-x^{k-a} \otimes x^{a} \otimes 1 G_{13} \\
v_{2, a}=x^{k-a} \otimes 1 \otimes x^{a} G_{12}-x^{a} \otimes x^{k-a} \otimes 1 G_{23}
\end{array}\right.
$$

We define increasing filtrations $\left\{\mathcal{V}_{i}\right\}$ and $\left\{\mathcal{M}_{i}\right\}, 0 \leq i \leq\left\lfloor\frac{k}{2}\right\rfloor$, on $E_{1}^{*}(2,1)$ and $E_{0}^{*}(2,1)$ respectively by

$$
\begin{aligned}
& \mathcal{V}_{i}=\bigoplus_{\substack{0 \leq a \leq i \\
\mathcal{M}_{-1}}}\left(\mathbf{V}_{a}+\mathbf{V}_{k-a}\right), \\
& \mathcal{M}_{i}=\mathcal{M}_{i-1} \bigoplus(2,1)-\text { isotypic component of } \bigoplus_{\begin{array}{r}
m-1 \geq a \geq b \geq c \geq 0 \\
a+b+c=m+k
\end{array}} \mathbb{Q}\left(\mathcal{S}_{3} x^{a} \otimes x^{b} \otimes x^{c}\right), \\
& \left.\mathcal{M}_{3} x^{m} \otimes x^{k-i} \otimes x^{i}\right)
\end{aligned}
$$

These filtrations are compatible with the symmetric structure and $d\left(\mathcal{V}_{i}\right)<\mathcal{M}_{i}$. We show that the differential induces an injective map between associated graded $\mathcal{S}_{3}$-modules: the matrix of the induced differential from $\mathcal{V}_{i} / \mathcal{V}_{i-1}$ to $\mathcal{M}_{i} / \mathcal{M}_{i-1}$ contains the following block given by the coordinates of $d v_{1, i}, d v_{2, i}, d v_{1, k-i}$ in the canonical basis $\left(x^{m} \otimes x^{k-i} \otimes x^{i}, x^{m} \otimes x^{i} \otimes x^{k-i}, x^{k-i} \otimes x^{m} \otimes x^{i}, \ldots\right)$

$$
\left(\begin{array}{rrr}
1 & 1 & 0 \\
-1 & 0 & 1 \\
1 & 1 & -1
\end{array}\right)
$$

hence the dimension of $d\left(\mathcal{V}_{i} / \mathcal{V}_{i-1}\right)$ is greater or equal to 3. By Schur lemma $\operatorname{dim} d\left(\mathcal{V}_{i} / \mathcal{V}_{i-1}\right)=4$, hence the "graded" differential is injective. In the very special case of an even $k, i=\frac{k}{2}$, the component $\mathcal{V}_{i} / \mathcal{V}_{i-1} \cong \mathbf{V}_{i}+\mathbf{V}_{k-i}$ has dimension 2, the differential is non-zero, hence injective.

Lemma 3.10. For $m \leq k \leq 2 m$, the dimension of the space of cocycles in the space $E_{1}^{(2 m-1)+2 k}(2,1)$ is at most two. 
Proof. In the proof we use the method of Lemmas 3.2 and 3.9. We will show that the differential is injective on a subspace of codimension two, $\mathcal{W}$, using an associated graded morphism. In the case $k=2 m, \mathcal{W}=0$ and the injectivity is obvious. Let us define the $V(2,1)$ submodules $\mathbf{W}_{a}, 0 \leq a \leq 2 m-1-k$, by

$$
\mathbf{W}_{a}:\left\{\begin{array}{l}
w_{1, a}=x^{m-a} \otimes 1 \otimes x^{k+a-m} G_{12}-x^{m-a} \otimes x^{k+a-m} \otimes 1 G_{13} \\
w_{2, a}=x^{m-a} \otimes 1 \otimes x^{k+a-m} G_{12}-x^{k+a-m} \otimes x^{m-a} \otimes 1 G_{23},
\end{array}\right.
$$

next the increasing filtrations $\mathcal{W}_{i}$ and $\mathcal{N}_{i}$ for $0 \leq i \leq\left\lfloor\frac{2 m-k}{2}\right\rfloor$ by

$$
\begin{aligned}
& \mathcal{W}_{0}=\mathbf{W}_{0}, \\
& \mathcal{W}_{i}=\mathbf{W}_{0} \oplus\left(\bigoplus_{1 \leq a \leq i}\left(\mathbf{W}_{a}+\mathbf{W}_{2 m-k-a}\right)\right), \\
& \mathcal{W}=\mathcal{W}_{\left\lfloor\frac{2 m-k}{2}\right\rfloor}, \\
& \mathcal{N}_{-1}=(2,1)^{2}-\text { isotypic component of } \underset{\substack{m \geq a \geq b \geq c \geq 0 \\
a+b+c=m+k}}{\bigoplus} \mathbb{Q}\left(\mathcal{S}_{3} x^{a} \otimes x^{b} \otimes x^{c}\right), \\
& \mathcal{N}_{i}=\mathcal{N}_{i-1} \oplus(2,1)-\text { isotypic component of } \mathbb{Q}\left(\mathcal{S}_{3} x^{m} \otimes x^{m-i} \otimes x^{k-m+i}\right) .
\end{aligned}
$$

The filtrations are compatible with the differential and the symmetric structure. The associated graded differential $\mathcal{W}_{i} / \mathcal{W}_{i-1} \rightarrow \mathcal{N}_{i} / \mathcal{N}_{i-1}$ is injective:

1) for $i=0, \mathcal{W}_{0} / \mathcal{W}_{-1}=\mathcal{W}_{0}$ is a simple module and $d\left(\mathcal{W}_{0}\right) \neq 0$

$2)$ for $i \geq 1$, the coordinates of $d\left(w_{1, i}\right), d\left(w_{2, i}\right), d\left(w_{1,2 m-k-i}\right)$ in the basis $\left(x^{m} \otimes\right.$ $\left.x^{m-i} \otimes x^{k-m+i}, x^{m} \otimes x^{k-m+i} \otimes x^{m-i}, x^{m-i} \otimes x^{m} \otimes x^{k-m+i}, \ldots\right)$ is given by the matrix in the proof of Lemma 3.9

$3)$ in the special case of an even $k$ and $2 i=2 m-k$, the component $\mathcal{W}_{i} / \mathcal{W}_{i-1}$ has dimension 2 and the differential $\mathcal{W}_{i} / \mathcal{W}_{i-1} \rightarrow \mathcal{N}_{i} / \mathcal{N}_{i-1}$ is non-zero, hence injective by Schur lemma.

Lemma 3.11. For $m \leq k \leq 2 m$, the space of cocycles in $E_{1}^{(2 m-1)+2 k}(2,1)$ is two dimensional and it coincides with the space of coboundaries.

Proof. This is a consequence of the previous lemma and of the fact that the differential $d: E_{2}^{2(2 m-1)+2 j}(2,1) \cong V(2,1) \rightarrow E_{1}^{(4 m-1)+2 j}(2,1)$ is non-zero for $0 \leq j \leq m$, hence the space of coboundaries has dimension two, by Schur lemma.

In the next two propositions, the term corresponding to $\left\lfloor\frac{3 m}{2}\right\rfloor$ and the term corresponding to $\left\lceil\frac{3 m}{2}\right\rceil$ should be taken only once for $m$ even.

Proposition 3.12. The Poincaré polynomial of the $(2,1)$ component of the cohomology $H^{*}\left(F\left(\mathbb{C} P^{m}, 3\right)\right)$ is given by:

$$
\begin{aligned}
P_{\left(F\left(\mathbb{C} P^{m}, 3\right)\right)(2,1)}(t, s)= & \sum_{k=1}^{m-1} 2 \mu_{2,1}(k) t^{2 k}+\sum_{k=m}^{\left\lfloor\frac{3 m}{2}\right\rfloor} 2\left(\mu_{2,1}(k)-k+m-1\right) t^{2 k}+ \\
& +\sum_{k=\left\lceil\frac{3 m}{2}\right\rceil}^{2 m-1} 2\left(\mu_{2,1}(3 m-k)-k+m-1\right) t^{2 k}+ \\
& +\sum_{k=2 m}^{3 m-1} 2\left(\mu_{2,1}(3 m-k)-3 m+k\right) t^{2 k}= \\
= & 2 t^{2}+4 t^{4}+6 t^{6}+10 t^{8}+\ldots+4 t^{6 m-10}+2 t^{6 m-8} .
\end{aligned}
$$

where the coefficients in the second expansion become stable for $m \geq 5$.

Proof. This is a direct consequence of dimensions counting for $E_{*}^{*}(2,1)$ in Section 2 and the previous three Lemmas. The coefficients in the second expansion are computed using Proposition 2.1 and the Appendix. 
Remark 3.13. For $m=2,3,4$ we have:

$$
\begin{aligned}
& P_{\left(F\left(\mathbb{C} P^{2}, 3\right)\right)(2,1)}(t, s)=2 t^{2}+2 t^{4} \\
& P_{\left(F\left(\mathbb{C} P^{3}, 3\right)\right)(2,1)}(t, s)=2 t^{2}+4 t^{4}+4 t^{6}+4 t^{8}+2 t^{10} \\
& P_{\left(F\left(\mathbb{C} P^{4}, 3\right)\right)(2,1)}(t, s)=2 t^{2}+4 t^{4}+6 t^{6}+8 t^{8}+8 t^{10}+6 t^{12}+4 t^{14}+2 t^{16}
\end{aligned}
$$

The contribution to the cohomology of the $(1,1,1)$ component is obvious from the table in Lemma 2.1

Proposition 3.14. The Poincaré polynomial for the $(1,1,1)$ component of the cohomology $H^{*}\left(F\left(\mathbb{C} P^{m}, 3\right)\right)$ is given by:

$$
\begin{aligned}
P_{\left(F\left(\mathbb{C} P^{m}, 3\right)\right)(1,1,1)}(t, s) & =\sum_{k=3}^{\left\lfloor\frac{3 m}{2}\right\rfloor} \mu_{1,1,1}(k) t^{2 k}+\sum_{k=\left\lceil\frac{3 m}{2}\right\rceil}^{3 m-3} \mu_{1,1,1}(3 m-k) t^{2 k}= \\
& =t^{6}+2 t^{8}+\ldots+2 t^{6 m-8^{2}}+t^{6 m-6} .
\end{aligned}
$$

Proof of Theorem 1.1. The Poincaré polynomial of $E_{q}^{*}$ is

$$
P_{E_{q}^{*}}(t, s)=\alpha_{q}\left(1+t^{2}+\ldots+t^{2 m}\right)^{3-q} t^{q(2 m-1)} s^{q},
$$

where $\sum_{i \geq 0} \alpha_{i} t^{i}=(1+t)(1+2 t)$ is the Poincaré polynomial of the Arnold algebra $\mathcal{A}(3) \cong H^{*}(F(\mathbb{C}, 3))$, see $[\mathrm{A}$. From the previous Lemmas the Poincaré polynomials of the spaces of cocycles in $E_{1}^{*}(3)$ and $E_{1}^{*}(2,1)$ are given by:

$$
\begin{aligned}
& P_{Z_{1}^{*}(3)}(t, s)=s\left(t^{4 m-1}+t^{4 m-3}+\ldots+t^{6 m-3}\right), \\
& P_{Z_{1}^{*}(2,1)}(t, s)=2 s\left(t^{4 m-1}+t^{4 m-3}+\ldots+t^{6 m-1}\right) .
\end{aligned}
$$

Thus we have

$$
\begin{aligned}
P_{F\left(\mathbb{C P} P^{m}, 3\right)}(t, s)= & \left(1+t^{2}+t^{4}+\ldots+t^{2 m}\right)^{3}-\left[3\left(1+t^{2}+t^{4}+\ldots+t^{2 m}\right)^{2} t^{2 m}-\right. \\
& \left.-\left(t^{4 m}+t^{4 m+2}+\ldots+t^{6 m-2}\right)-2\left(t^{4 m}+t^{4 m+2}+\ldots+t^{6 m}\right)\right] \\
& +s\left(t^{4 m-1}+t^{4 m+1}+\ldots+t^{6 m-3}\right),
\end{aligned}
$$

and a straightforward simplification gives the result.

In Section 1 the $(k, q)$ supports of $E_{*}^{*}$ and $H_{*}^{*}$ are drawn for each of the three types of $\mathcal{S}_{3}$-modules and for the stable cases: $m \geq 5$.

\section{Algebra structure of the cohomology}

The configuration space $F\left(\mathbb{C} P^{m}, 3\right)$ is the total space in two natural fibrations:

$$
\begin{aligned}
& {\stackrel{\circ}{C} P^{m}}^{\rightarrow} F\left(\mathbb{C} P^{m}, 3\right) \rightarrow F\left(\mathbb{C} P^{m}, 2\right), \\
& F\left(\stackrel{\circ}{\mathbb{C} P} P^{m}, 2\right) \hookrightarrow F\left(\mathbb{C} P^{m}, 3\right) \rightarrow \mathbb{C} P^{m},
\end{aligned}
$$

see [FN]. If $X$ is a connected manifold, $\stackrel{\circ}{X}$ and $\stackrel{\circ}{X}$ denote the spaces $X \backslash\{$ one point $\}$ and $X \backslash$ two points $\}$ respectively. For $m=3$ and $m=4$ the spectral sequences associated to these fibrations are used in $[\mathrm{S}$ to reduce long computations with the Križ model. Using the Poincaré polynomial of $F\left(\mathbb{C} P^{m}, 3\right)$, Theorem 1.1, we can describe the structure of the Serre spectral sequences corresponding to these fibrations, proving some conjectures from $[\underline{S}$. The terms of spectral sequences will be written in bold characters $\mathbf{E}_{*}^{* * *}$. We will use the notation $r_{m}(x, y)=x^{m}+$ $x^{m-1} y+\ldots+y^{m}$ and $C_{m}(t)=r_{m}\left(1, t^{2}\right)=1+t^{2}+\ldots+t^{2 m}$. The cohomology of the fibers are given in the next lemma: 
Lemma 4.1. a) The cohomology algebra of $\stackrel{\circ}{\mathbb{C}} P^{m}$ has the presentation:

$$
H^{*}\left(\stackrel{\circ}{\mathbb{C} P} P^{m}\right) \cong \mathbb{Q}\left\langle y, z \mid y^{m}, y z\right\rangle
$$

with $|y|=2,|z|=2 m-1$. Its Poincaré polynomial is given by

$$
P_{\stackrel{C}{ } \stackrel{\circ}{m}}(t)=C_{m-1}(t)+t^{2 m-1} \text {. }
$$

b) The cohomology algebra of $F\left(\stackrel{\circ}{\mathbb{C} P} P^{m}, 2\right)$ has the presentation:

$$
H^{*}\left(F\left(\stackrel{\circ}{\mathbb{C} P}{ }^{m}, 2\right)\right) \cong \mathbb{C}\left\langle y, z, u \mid y^{m}, z^{m}, r_{m}(y, z), y u, z u\right\rangle
$$

with $|y|=|z|=2,|u|=4 m-3$. Its Poincaré polynomial is given by

$$
\begin{aligned}
& P_{F(\mathbb{C} P m, 2)}(t)=1+2 t^{2}+3 t^{4}+\ldots+m t^{2 m-2}+(m-2) t^{2 m}+ \\
& +(m-3) t^{2 m+2}+\ldots+2 t^{4 m-8}+t^{4 m-6}+t^{4 m-3} \text {. }
\end{aligned}
$$

Proof. a) Obviously $\stackrel{\circ}{\mathbb{C}} P^{m} \simeq \mathbb{C} P^{m-1}$ and the Mayer-Vietoris sequence for the decomposition $\stackrel{\circ}{\mathbb{C}} P^{m}=\stackrel{\circ}{\mathbb{C}} P^{m} \cup D^{2 m}$ gives the Betti numbers and the algebra morphism $H^{*}\left(\stackrel{\circ}{\mathbb{C}} P^{m}\right) \rightarrow H^{*}\left(\stackrel{\circ}{\mathbb{C}} P^{m}\right)$, injective for $* \leq 2 m-2$.

b) For these computation we use the version of the Križ model for a punctured complex projective manifold $\stackrel{\circ}{X}$ introduced in $\mathrm{BMP}(X$ is simply connected and the coefficients should be complex): the fundamental class $\omega$ becomes 0 . Denote by $y, z$ and $u$ the cohomology classes of $x \otimes 1,1 \otimes x$ and $x^{m-1} \otimes 1 G_{12}$ respectively. The equality $x^{m}=0$ in the model implies $y^{m}=z^{m}=0$ and also $y u=z u=0$; the relation $r_{m}(y, z)=0$ is a consequence of $d G_{12}=y^{m-1} z+\ldots+y z^{m-1}$ and of the relations $y^{m}=z^{m}=0$. A basis for the cohomology is

$$
\left\{1 ; y, z ; y^{2}, y z, z^{2} ; \ldots ; y^{m-1}, y^{m-2} z, \ldots, z^{m-1} ; y^{m-1} z, \ldots, y^{2} z^{m-2} ; \ldots ; y^{m-1} z^{m-2} ; u\right\} .
$$

We show that the two spectral sequences have a nontrivial differential at a unique place: for the first fibration, the "first" differential, $d_{2}$, and for the second one, the "last" differential, $d_{2 m}$, are non zero.

Proposition 4.2. The Serre spectral sequence of the fibration

$$
\stackrel{\circ}{\mathbb{C}} P^{m} \hookrightarrow F\left(\mathbb{C} P^{m}, 3\right) \rightarrow F\left(\mathbb{C} P^{m}, 2\right)
$$

collapses at $\mathbf{E}_{3}^{*, *}$.

Proof. The cohomology algebra of the base is a simple consequence of the Križ model (one can see [S], Theorem 1,2): its Poincaré polynomial is:

$$
P_{F\left(\mathbb{C P} P^{m}, 2\right)}(t)=C_{m-1}(t) C_{m}(t) .
$$

All the differentials in the lower rectangle $(4 m-2) \times(2 m-2)$ are zero for degree reason. From 1.1 the $(6 m-5)^{t h}$ Betti number of $F\left(\mathbb{C} P^{m}, 3\right)$ equals 1 , therefore $\mathbf{E}_{2}^{4 m-4,2 m-1}$ should contain a cocycle, and this implies that

$$
d_{2}(1 \otimes z)=(\lambda x \otimes 1+\mu 1 \otimes x) \otimes y^{m-1},
$$

where at least one of the complex numbers $\lambda, \mu$ is non zero. As a consequence, the differential

$$
d_{2}: \mathbf{E}_{2}^{i, 2 m-1} \rightarrow \mathbf{E}_{2}^{i+2,2 m-2}
$$


is injective for $i=0, \ldots, 2 m-2$ and surjective for $i=2 m-2, \ldots, 4 m-2$ because the matrix of $d_{2}$ with respect to the bases $\left\{x^{j} \otimes x^{i-j} \otimes z\right\}$ and $\left\{x^{j} \otimes x^{i-j} \otimes y^{m-1}\right\}$ contains two triangular blocks, at least one of maximal rank:

$$
\left(\begin{array}{ccc}
\lambda & & 0 \\
& \cdots & \\
* & & \lambda
\end{array}\right) \text { and }\left(\begin{array}{ccc}
\mu & & * \\
& \cdots & \\
0 & & \mu
\end{array}\right) \text {. }
$$

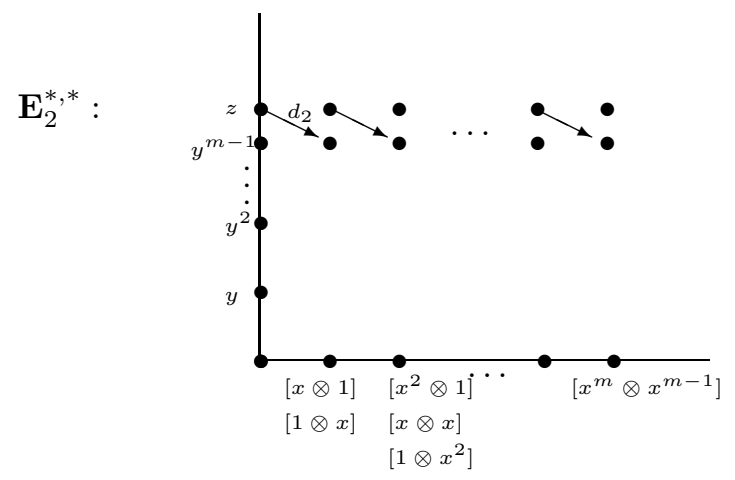

Computing the Poincaré polynomial of the term $\mathbf{E}_{3}^{*, *}$ we find

$$
\begin{aligned}
P_{\mathbf{E}_{3}^{* * *}}(t) & =C_{m-2}(t) C_{m-1}(t) C_{m}(t)+t^{2 m-2} C_{m-1}(t)+t^{4 m-1} C_{m-1}(t)= \\
& =C_{m-1}^{3}+t^{4 m-1} C_{m-1}(t),
\end{aligned}
$$

the same with $P_{F\left(\mathbb{C} P^{m}, 3\right)}(t)$.

Proposition 4.3. The Serre spectral sequence of the fibration

$$
F\left(\stackrel{\circ}{\mathbb{C}} P^{m}, 2\right) \hookrightarrow F\left(\mathbb{C} P^{m}, 3\right) \rightarrow \mathbb{C} P^{m}
$$

has a unique non-zero differential $d_{2 m}: \mathbf{E}_{2 m}^{0,4 m-3} \rightarrow \mathbf{E}_{2 m}^{2 m, 2 m-2}$.

Proof. In the proof we will use complex coefficients (necessary for the given presentation of $\left.H^{*}\left(F\left(\stackrel{\circ}{\mathbb{C} P} P^{m}, 2\right) ; \mathbb{C}\right)\right)$; the structure of the differentials in the spectral sequence with complex coefficients gives the same structure for the rational spectral sequence.

Like in the previous proof, non zero differentials could appear only on the top horizontal line $\mathbf{E}_{*}^{*}, 4 m-3$, the remaining classes having even degrees. Its first Poincaré polynomial is

$$
P_{\mathbf{E}_{2}^{*, 4 m-3}}(t)=t^{4 m-3}+t^{4 m-1}\left(1+t^{2}+t^{4}+\ldots+t^{2 m-2}\right),
$$

whose second part equals the Poincaré polynomial of the odd cohomology of the space $F\left(\mathbb{C} P^{m}, 3\right)$. Therefore $1 \otimes u \in \mathbf{E}_{*}^{0,4 m-3}$ has a non zero differential and $x^{k} \otimes u$ must be a cocycle for $k=1,2, \ldots, m$. 


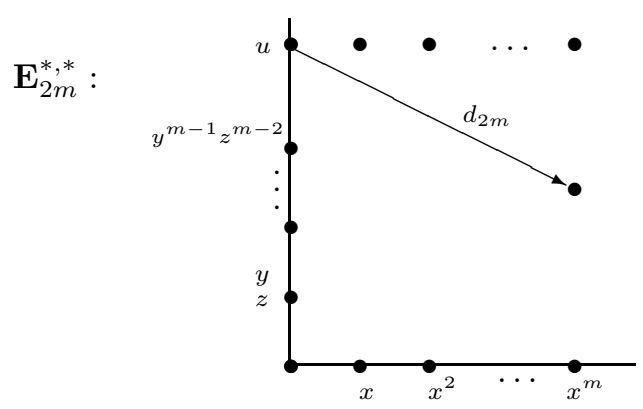

All these imply that

$d_{2}=\ldots=d_{2 m-1}=0, d_{2 m}(1 \otimes u)=x^{m} \otimes\left(c_{0} y^{m-1}+c_{1} y^{m-2} z+\ldots+c_{m-1} z^{m-1}\right) \neq 0$, and also that $d_{2 m+1}=d_{2 m+2}=\ldots=0$.

In the second part of this section we give a presentation of the cohomology algebras of the ordered and unordered configuration spaces.

Lemma 4.4. The subalgebra of cocycles $Z_{*}^{*}\left(\mathbb{C} P^{m}, 3\right)$ in $E_{*}^{*}\left(\mathbb{C} P^{m}, 3\right)$ has the presentation (as a graded commutative algebra):

$$
\begin{aligned}
& \left\langle\begin{array}{c|l}
a_{1}, a_{2}, a_{3}, & a_{i}^{m+1}, s_{m} w,\left(a_{i}-a_{k}\right) v_{j}, v_{1} v_{2}, v_{j} w, \\
w, v_{1}, v_{2} & \left(s_{1}^{2}-3 s_{2}\right) w,\left(s_{1} s_{2}-3 s_{3}\right) w,
\end{array}\left(\begin{array}{c}
a_{1} \\
a_{2} \\
a_{3}
\end{array}\right)=A\left(\begin{array}{c}
w \\
v_{1} \\
v_{2}
\end{array}\right)\right\rangle \\
& \text { where }\left|a_{i}\right|=2,|w|=\left|v_{j}\right|=4 m-1, s_{k}=a_{1}^{k}+a_{2}^{k}+a_{3}^{k} \text { and } A=\left(\begin{array}{ccc}
s_{1} / 3 & -a_{1} & 2 a_{1} \\
s_{1} / 3 & 2 a_{1} & -a_{1} \\
s_{1} / 3 & -a_{1} & -a_{1}
\end{array}\right) .
\end{aligned}
$$

Proof. From Lemmas 3.4 3.5 and 3.11 the algebra of cocycles has three generators $x \otimes 1 \otimes 1,1 \otimes x \otimes 1,1 \otimes 1 \otimes x$ in degree two and three other generators in higher degrees, $W \in E_{1}^{4 m-1}(3)$ and $V_{1}, V_{2} \in E_{1}^{4 m-1}(2,1)$, where

$$
\begin{aligned}
V_{1}= & \left(x^{m} \otimes 1 \otimes 1+x^{m-1} \otimes x \otimes 1+\ldots+1 \otimes x^{m} \otimes 1\right) G_{13}- \\
& -\left(x^{m} \otimes 1 \otimes 1+x^{m-1} \otimes 1 \otimes x+\ldots+1 \otimes 1 \otimes x^{m}\right) G_{12}, \\
V_{2}= & \left(x^{m} \otimes 1 \otimes 1+x^{m-1} \otimes x \otimes 1+\ldots+1 \otimes x^{m} \otimes 1\right) G_{23}- \\
& -\left(x^{m} \otimes 1 \otimes 1+x^{m-1} \otimes 1 \otimes x+\ldots+1 \otimes 1 \otimes x^{m}\right) G_{12} .
\end{aligned}
$$

From the algebra $\mathcal{B}$ with the above presentation, we define a map $\theta: \mathcal{B} \rightarrow Z_{*}^{*}$ by

$$
a_{i} \mapsto p_{i}^{*}(x), w \mapsto W, v_{j} \mapsto V_{j},
$$

and this is well defined because:

1) $x^{m+1}=0$ implies $\theta\left(a_{i}^{m+1}\right)=0$ and, from Remark 3.6, $\theta\left(s_{m} w\right)=t_{m} W=0$;

2) for every $i=1,2,3$, the product $p_{i}^{*}(x) V_{1}$ equals

$$
\left(x^{m} \otimes x \otimes 1+\ldots+x \otimes x^{m} \otimes 1\right) G_{13}-\left(x^{m} \otimes 1 \otimes x+\ldots+x \otimes 1 \otimes x^{m}\right) G_{12} ;
$$

3) the cocycles $W V_{i}$ and $V_{1} V_{2}$ must be zero due to injectivity of $d: E_{2}^{*} \rightarrow E_{1}^{*}$;

4) the polynomials $t_{1}^{2} W$ and $3 t_{2} W$ are symmetric, so it is enough to compute the coefficients of $G_{12}$ : we find the same value

$(18 m-36) x^{m} \otimes 1 \otimes x^{2}+(18 m-63) x^{m-1} \otimes 1 \otimes x^{3}+\ldots-(9 m-18) x^{2} \otimes 1 \otimes x^{m}$, and in a similar way, the coefficients of $G_{12}$ in $t_{1} t_{2} W$ and $3 t_{3} W$ coincide:

$$
(18 m-54) x^{m} \otimes 1 \otimes x^{3}+(18 m-81) x^{m-1} \otimes 1 \otimes x^{4}+\ldots-(9 m-27) x^{3} \otimes 1 \otimes x^{m} ;
$$


5) a basis of the three dimensional space $Z_{1}^{4 m+1}$ is given by the cocycles $t_{1} W$, $p_{1}^{*}(x) V_{j}(j=1,2)$. The formulae

$$
\begin{aligned}
& p_{1}^{*}(x) W=1 / 3 t_{1} W-p_{1}^{*}(x) V_{1}+2 p_{1}^{*}(x) V_{2}, \\
& p_{2}^{*}(x) W=1 / 3 t_{1} W+2 p_{1}^{*}(x) V_{1}-p_{1}^{*}(x) V_{2}, \\
& p_{3}^{*}(x) W=1 / 3 t_{1} W-p_{1}^{*}(x) V_{1}-p_{1}^{*}(x) V_{2}
\end{aligned}
$$

are obtained by direct computations.

The map $\theta$ is surjective because the generators $\left\{p_{i}^{*}(x)\right\}_{i=1,2,3},\left\{V_{j}\right\}_{j=1,2}$ and $W$ belong to the image. The restriction of $\theta$ to $\mathcal{B}_{0}$, the subalgebra generated by $\left\{a_{i}\right\}_{i=1,2,3}$, is injective. The defining relations of $\mathcal{B}$ show that each homogenous component of $\mathcal{B}_{1}$, the ideal generated by the elements $w,\left\{v_{j}\right\}_{j=1,2}$ is the span of the elements $s_{1}^{q} w, a_{1}^{q} v_{1}, a_{1}^{q} v_{2}$; therefore $3 \geq \operatorname{dim} \mathcal{B}_{1}^{*} \geq \operatorname{dim} Z_{1}^{*}=3$ (with one exception: $2 \geq \operatorname{dim} \mathcal{B}_{1}^{6 m-1} \geq \operatorname{dim} Z_{1}^{6 m-1}=2$ ) and $\theta$ is an isomorphism.

Proof of Theorem 1.3. Let us denote by $\mathcal{A}$ the graded commutative algebra

$$
\left\langle\alpha_{1}, \alpha_{2}, \alpha_{3}, \eta \mid \alpha_{1}^{m+1}, r_{m}\left(\alpha_{i}, \alpha_{j}\right),\left(\alpha_{i}-\alpha_{j}\right) \eta, \alpha_{1}^{m} \eta\right\rangle
$$

where $\left|\alpha_{i}\right|=2$ and $|\eta|=4 m-1$. We define the algebra morphisms $\varphi, \psi_{0}$ and $\psi$

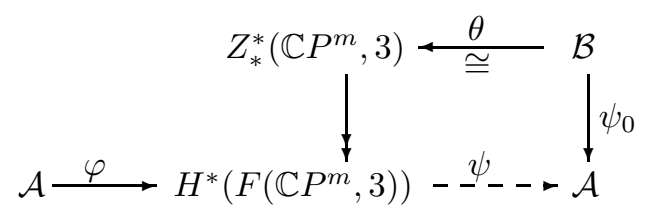

and we show that $\varphi$ is surjective and $\psi \varphi=i d_{\mathcal{A}}$.

The morphism $\varphi$ given by $\alpha_{i} \mapsto\left[p_{i}^{*}(x)\right], \eta \mapsto[W]$ is well defined:

1) $x^{m+1}=0$ implies $\varphi\left(\alpha_{i}^{m+1}\right)=0$;

2) we have $\varphi\left(r_{m}\left(\alpha_{i}, \alpha_{j}\right)\right)=\left[d G_{i j}\right]$;

3) $\varphi\left(\left(\alpha_{1}-\alpha_{2}\right) \eta\right)=0$ is a consequence of the following computation

$$
\begin{aligned}
(x \otimes 1 \otimes 1-1 \otimes x \otimes 1) W & =3\left(x^{m} \otimes x \otimes 1+\ldots+x \otimes x^{m} \otimes 1\right)\left(G_{23}-G_{13}\right) \\
& =d\left(3(1 \otimes x \otimes 1) G_{13} G_{23}\right)
\end{aligned}
$$

and similarly for the relation $\varphi\left(\left(\alpha_{1}-\alpha_{3}\right) \eta\right)=0$;

4) $\varphi\left(\alpha_{1}^{m} \eta\right)=\left[x^{m} \otimes 1 \otimes 1\right][W]$ and this product is represented by the coboundary

$$
\begin{aligned}
\left(x^{m} \otimes 1 \otimes 1\right) W & =x^{m} \otimes x^{m} \otimes 1\left(2 m G_{23}-m G_{13}\right)-m\left(x^{m} \otimes 1 \otimes x^{m}\right) G_{12}= \\
& =d\left(m\left(x^{m} \otimes 1 \otimes 1\right)\left(2 G_{12} G_{23}-G_{12} G_{13}\right)\right) .
\end{aligned}
$$

The even part of the cohomology is generated by $\left[p_{i}^{*}(x)\right]_{i=1,2,3}$ and its odd part by $\left[t_{k} W\right]$, see Lemmas 3.5 and 3.11, therefore $\varphi$ is surjective.

The algebra morphism $\psi_{0}: \mathcal{B} \rightarrow \mathcal{A}$ given by $a_{i} \mapsto \alpha_{i}, w \mapsto \eta, v_{j} \mapsto 0$ is well defined:

1) $\psi_{0}\left(a_{1}^{m+1}\right)=\alpha_{1}^{m+1}=0$ and the relations $\psi_{0}\left(a_{i}^{m+1}\right)=0, i=2,3$ are consequences of $\alpha_{1}^{m+1}=0$ and $\left(\alpha_{1}-\alpha_{i}\right) r_{m}\left(\alpha_{1}, \alpha_{i}\right)=0$;

2) $\psi_{0}\left(s_{m} w\right)=0$ is a consequence of $\alpha_{i}^{m} \eta=0$;

3 ) the relations containing only monomials in $v_{1}, v_{2}$ are obviously sent to zero;

4) the relation $\left(s_{1}^{2}-3 s_{2}\right) w$ is sent to $\left(\tau_{1}^{2}-3 \tau_{2}\right) \eta=\left(\left(3 \alpha_{1}\right)^{2}-9 \alpha_{1}^{2}\right) \eta=0$ (where $\left.\tau_{k}=\alpha_{1}^{k}+\alpha_{2}^{k}+\alpha_{3}^{k}\right)$, and similarly for $\left(s_{1} s_{2}-3 s_{3}\right) w$; 
5) the last three relations in $\mathcal{B}$ are sent to zero because $v_{j} \mapsto 0$ and $\alpha_{1} \eta=\frac{1}{3} \tau_{1} \eta$. The morphism $\psi_{0}$ annihilates the inverse image of the ideal of coboundaries in $Z_{*}^{*}$, hence it induces the algebra morphism $\psi: H^{*} \rightarrow \mathcal{A}$ :

1) $\psi_{0}\left(\theta^{-1}\left(d G_{i j}\right)\right)=r_{m}\left(\alpha_{i}, \alpha_{j}\right)=0$

2) from Lemma 3.11 we have $\psi_{0}\left(\theta^{-1}\left(d E_{2}^{*}\right)\right)=\psi_{0}\left(\theta^{-1}\left(E_{0}^{*}\left(V_{1}, V_{2}\right)\right)\right)=0$.

Finally, the composition $\psi \varphi$ leaves fixed the generators of $\mathcal{A}$, hence $\psi \varphi=i d$.

In the next lemmas and their proofs the rational polynomials $P_{k}$ are defined in Section [1] if $T_{k}=X_{1}^{k}+X_{2}^{k}+X_{3}^{k}$, then $T_{k}=P_{k}\left(T_{1}, T_{2}, T_{3}\right)$, and $\sigma_{1}, \sigma_{2}, \sigma_{3}$ are the Viète polynomials expressed in terms of Newton polynomials:

$$
\sigma_{1}=T_{1}, \quad \sigma_{2}=\frac{1}{2}\left(T_{1}^{2}-T_{2}\right), \quad \sigma_{3}=\frac{1}{6} T_{1}^{3}-\frac{1}{2} T_{1} T_{2}+\frac{1}{3} T_{3} .
$$

Lemma 4.5. The algebra of $\mathcal{S}_{3}$-invariant cocycles has the presentation:

$$
\left\langle\tau_{1}, \tau_{2}, \tau_{3}, \eta \mid P_{m+1}, P_{m+2}, P_{m+3},\left(\tau_{1}^{2}-3 \tau_{2}\right) \eta,\left(\tau_{1} \tau_{2}-3 \tau_{3}\right) \eta, P_{m} \eta\right\rangle
$$

where $\left|\tau_{i}\right|=2 i(i=1,2,3),|\eta|=4 m-1$ and $P_{k}=P_{k}\left(\tau_{1}, \tau_{2}, \tau_{3}\right)$.

Proof. As in the proof of Lemma 4.4 we define a surjective algebra morphism $\theta$ between the algebra $\mathcal{C}$ given by the above presentation and $Z_{*}^{*}(3)$ :

$$
\theta: \mathcal{C} \longrightarrow Z_{*}^{*}(3), \quad \tau_{i} \mapsto t_{i}, i=1,2,3, \eta \mapsto W .
$$

The element $\theta\left(P_{k}\left(\tau_{1}, \tau_{2}, \tau_{3}\right)\right)=t_{k}$ is zero in $Z_{*}^{*}(3)$ for $k \geq m+1$ and the images of the last three relations are zero from the steps 1 and 4 in the proof of Lemma 4.4 . The Newton symmetric polynomials $t_{1}, t_{2}, t_{3}$ generate $Z_{0}^{*}(3)$ and, from Lemma 3.5 , $Z_{1}^{*}(3)$ is generated by $t_{1}, t_{2}, t_{3}$ and $W$, hence $\theta$ is surjective. We denote by $\mathcal{C}_{0}$ the subalgebra generated by $\tau_{1}, \tau_{2}, \tau_{3}$ and by $\mathcal{C}_{1}$ the ideal generated by $\eta$. We have

$$
1 \geq \operatorname{dim} \mathcal{C}_{1}^{k} \geq \operatorname{dim} Z_{1}^{k}(3)=1 \text { for } k=4 m-1,4 m+1, \ldots, 6 m-3
$$

due to $Q\left(\tau_{1}, \tau_{2}, \tau_{3}\right) \eta=Q\left(\tau_{1}, \frac{1}{3} \tau_{1}^{2}, \frac{1}{9} \tau_{1}^{3}\right) \eta$; this relation and $P_{m} \eta=0$ imply

$$
0=\operatorname{dim} \mathcal{C}_{1}^{6 m-1}=\operatorname{dim} Z_{1}^{6 m-1} .
$$

Now we construct an inverse of the restriction map $\left.\theta\right|_{\mathcal{C}_{0}}$ : the algebra map

$$
\varepsilon: \operatorname{Symm}\left[X_{1}, X_{2}, X_{3}\right]=\mathbb{Q}\left[T_{1}, T_{2}, T_{3}\right] \rightarrow \mathcal{C}_{0} \text { given by } T_{i} \mapsto \tau_{i}
$$

induces a map $Z_{0}^{*}(3) \rightarrow \mathcal{C}_{0}$ because $\varepsilon$ is zero on the sums $Q_{a, b, c}=\sum_{\pi \in \mathcal{S}_{3}} \pi\left(X_{1}^{a} X_{2}^{b} X_{3}^{c}\right)$ $(a \geq b \geq c \geq 0)$ if $a \geq m+1$ :

1) first $P_{m+1}=P_{m+2}=P_{m+3}=0$ in $\mathcal{C}$ implies that $P_{k}=\sigma_{1} P_{k-1}-\sigma_{2} P_{k-2}+$ $\sigma_{3} P_{k-3}$ is also zero in $\mathcal{C}$ for any $k \geq m+4$;

2) secondly, $Q_{a, b, 0}=P_{a} P_{b}-P_{a+b}$ and

$$
Q_{a, b, c}=P_{a} P_{b} P_{c}-\left(P_{a+b} P_{c}+P_{a+c} P_{b}+P_{a} P_{b+c}+P_{a+b+c}\right) .
$$

Lemma 4.6. The vector space $E_{1}^{2 k+2 m-1}(3)$ is generated by

$$
\Gamma_{k}=x^{k} \otimes 1 \otimes 1 G_{12}+x^{k} \otimes 1 \otimes 1 G_{13}+1 \otimes x^{k} \otimes 1 G_{23} \text { and } M\left(t_{1}, t_{2}, t_{3}\right) \Gamma_{0},
$$

where $M$ is an arbitrary monomial of degree $2 k$. 
Proof. By induction on $k$ : for $k=0$ we have $E_{1}^{2 m-1}(3)=\mathbb{Q}\left\langle\Gamma_{0}\right\rangle$. The canonical basis in $E_{1}^{2 k+2 m-1}(3)$ is given by

$$
\left\{B_{i, j}=x^{i} \otimes 1 \otimes x^{j} G_{12}+x^{i} \otimes x^{j} \otimes 1 G_{13}+x^{j} \otimes x^{i} \otimes 1 G_{23}\right\}_{i+j=k} .
$$

Now we start an induction on $j$ : if $(i, j)=(k, 0)$ we have $\Gamma_{k}$; if $j \geq 1$ we find that

$$
B_{i, j}=t_{1} B_{i, j-1}-2 B_{i+1, j-1}
$$

is a linear combination of $\Gamma_{k}$ and $M\left(t_{1}, t_{2}, t_{3}\right) \Gamma_{0}$.

Proof of Theorem 1.4 This is similar to the proof of Theorem 1.3 in the next diagram $\mathcal{D}$ is the algebra presented in the statement of Theorem 1.4 and $\mathcal{C}$ and $\theta$ are from Lemma 4.5 .

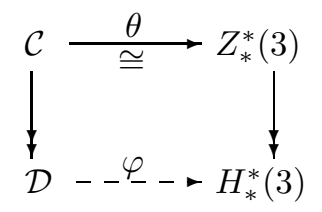

The induced algebra morphism $\varphi: \tau_{i} \mapsto\left[t_{i}\right], i=1,2,3, \eta \mapsto[W]$ is an isomorphism because the ideal generated by $\eta$ is isomorphic to $Z_{1}^{*}(3) \cong H_{1}^{*}(3)$ (see Lemma 4.5), and the subalgebra generated by $\tau_{1}, \tau_{2}, \tau_{3}$ is isomorphic to $H_{0}^{*}(3) \cong Z_{0}^{*}(3) / B_{0}^{*}(3)$ : the preimage of coboudaries in $Z_{*}^{*}(3)$ is generated by the three relations containing quadratic terms in $P_{*}$, as a consequence of Lemma 4.6 and of the following relations:

$$
\begin{aligned}
d\left(\Gamma_{0}\right) & =\theta\left(P_{0} P_{m}+P_{1} P_{m-1}+\ldots+P_{m} P_{0}-(m+1) P_{m}\right), \\
d\left(\Gamma_{1}\right) & =\theta\left(P_{1} P_{m}+P_{2} P_{m-1}+\ldots+P_{m} P_{1}\right), \\
d\left(\Gamma_{2}\right) & =\theta\left(P_{2} P_{m}+P_{3} P_{m-1}+\ldots+P_{m} P_{2}\right), \\
d\left(M\left(t_{1}, t_{2}, t_{3}\right) \Gamma_{0}\right) & =M\left(t_{1}, t_{2}, t_{3}\right) d\left(\Gamma_{0}\right), \\
d\left(\Gamma_{k}\right) & =d\left(\sigma_{1} \Gamma_{k-1}-\sigma_{2} \Gamma_{k-2}+\sigma_{3} \Gamma_{k-3}\right)= \\
& \left.=\sigma_{1} d\left(\Gamma_{k-1}\right)-\sigma_{2} d\left(\Gamma_{k-2}\right)+\sigma_{3} d\left(\Gamma_{k-3}\right)\right) .
\end{aligned}
$$

For $k=4$, in the last relations we have to use also the relation $\sigma_{3} P_{m}=0$, and this is a consequence of $P_{k}=0$ for $k=m+1, m+2, m+3$.

\section{The Stable cohomology Classes}

For the infinite dimensional complex projective space we will consider configurations with an arbitrary number of points.

Theorem 5.1. The inclusion map

$$
\iota: F\left(\mathbb{C} P^{\infty}, n\right) \hookrightarrow\left(\mathbb{C} P^{\infty}\right)^{n}
$$

is a homotopy equivalence.

Proof. We define the homotopy inverse of the inclusion map $\iota$ by

$$
f:\left(\mathbb{C} P^{\infty}\right)^{n} \rightarrow F\left(\mathbb{C} P^{\infty}, n\right),\left(P_{0}, P_{1}, \ldots, P_{n-1}\right) \mapsto\left(Q_{0}, Q_{1}, \ldots, Q_{n-1}\right),
$$

where the coordinates of $Q_{j}=\left[q_{0}^{j}: q_{1}^{j}: \ldots\right]$ depend only on the coordinates of $P_{j}=\left[p_{0}^{j}: p_{1}^{j}: \ldots\right]$ :

$$
q_{n i+j}^{j}=p_{i}^{j} \text { and } q_{i}^{j}=0 \text { for the rest of coordinates. }
$$


For instance, if $n=3$, the triple $\left(P_{0}, P_{1}, P_{2}\right)$ (of not necessarily distinct points) is sent to the triple of distinct points $\left(Q_{0}, Q_{1}, Q_{2}\right)$ :

$$
\begin{aligned}
& P_{0}=\left[p_{0}^{0}: p_{1}^{0}: p_{2}^{0}: \ldots\right] \\
& P_{1}=\left[p_{0}^{1}: p_{1}^{1}: p_{2}^{1}: \ldots\right] \\
& P_{2}=\left[p_{0}^{2}: p_{1}^{2}: p_{2}^{2}: \ldots\right]
\end{aligned} \quad \mapsto \begin{aligned}
& Q_{0}=\left[p_{0}^{0}: 0: 0: p_{1}^{0}: 0: 0: p_{2}^{0}: 0: 0: \ldots\right] \\
& Q_{1}=\left[0: p_{0}^{1}: 0: 0: p_{1}^{1}: 0: 0: p_{2}^{1}: 0: \ldots\right] \\
& Q_{2}=\left[0: 0: p_{0}^{2}: 0: 0: p_{1}^{2}: 0: 0: p_{2}^{2}: \ldots\right]
\end{aligned}
$$

The homotopy is given by $H:\left(\mathbb{C} P^{\infty}\right)^{n} \times I \rightarrow\left(\mathbb{C} P^{\infty}\right)^{n}$,

$$
\left(\left(P_{0}, \ldots, P_{n-1}\right), t\right) \mapsto\left((1-t) P_{0}+t Q_{0}, \ldots,(1-t) P_{n-1}+t Q_{n-1}\right),
$$

where, for $P=\left[p_{0}: p_{1}: \ldots\right] \in \mathbb{C} P^{\infty}$ and $Q=\left[q_{0}: q_{1}: \ldots\right]$ with $q_{i}=$ linear form in $p_{0}, p_{1}, \ldots$, the point $(1-t) P+t Q$ is defined as $\left[(1-t) p_{0}+t q_{0}:(1-t) p_{1}+t q_{1}\right.$ : ...]; this makes sense in our case because the last non-zero entry $p_{i}$ gives a last non-zero entry in $(1-t) P+t Q$. The map $H$ is continuous on finite skeleta of $\left(\mathbb{C} P^{\infty}\right)^{n} \times I$ and its restriction $h$ to $F\left(\mathbb{C} P^{\infty}, n\right) \times I$ takes values in $F\left(\mathbb{C} P^{\infty}, n\right)$ : in fact we have $H\left(\left(\mathbb{C} P^{\infty}\right)^{n} \times(0,1]\right) \subset F\left(\mathbb{C} P^{\infty}, n\right)$. Obviously $\left.H\right|_{t=0}=i d=\left.h\right|_{t=0}$, $\left.H\right|_{t=1}=\iota \circ f,\left.h\right|_{t=1}=f \circ \iota$.

Corollary 5.2. The induced action of the symmetric group $\mathcal{S}_{n}$ on the cohomology algebra of the configuration space $F\left(\mathbb{C} P^{\infty}, n\right)$ is the natural action on the polynomial $\operatorname{ring} \mathbb{Q}\left[x_{1}, \ldots x_{n}\right]$.

Proof. The inclusion $F\left(\mathbb{C} P^{\infty}, n\right) \hookrightarrow\left(\mathbb{C} P^{\infty}\right)^{n}$ is $\mathcal{S}_{n}$-equivariant and induces an isomorphism in cohomology.

Proof of Theorem 1.5. Clear from 5.1 and 5.2. It is also clear that the first isomorphism is true with coefficients in $\mathbb{Z}$.

Proof of Corollary 1.6. Using [T, the image of the map $i^{*}: H^{*}\left(X^{n}\right) \rightarrow H^{*}(F(X, n))$ is contained in $H_{0}^{*}(F(X, n))$. For the space $\mathbb{C} P^{m}$, the first non zero coboundaries are $d\left(E_{1}^{2 m-1}\right)<E_{0}^{2 m}$. In the range $k \in\{0,1, \ldots, 2 m-1\}$ we have

$$
\begin{aligned}
H_{*}^{k}\left(F\left(\mathbb{C} P^{m}, n\right)\right) & =H_{0}^{k}\left(F\left(\mathbb{C} P^{m}, n\right)\right) \cong E_{0}^{k}\left(F\left(\mathbb{C} P^{m}, n\right)\right) \cong H^{k}\left(\left(\mathbb{C} P^{m}\right)^{n}\right) \\
& \cong H^{k}\left(\left(\mathbb{C} P^{\infty}\right)^{n}\right) \cong H^{k}\left(F\left(\mathbb{C} P^{\infty}, n\right)\right) .
\end{aligned}
$$

\section{Configurations of COllinear And NON COllinear POints}

For dimensions $2 \leq m \leq \infty$ the 3 -point configuration space $F\left(\mathbb{C} P^{m}, 3\right)$ splits into two parts:

$$
F\left(\mathbb{C} P^{m}, 3\right)=F_{c}\left(\mathbb{C} P^{m}, 3\right) \bigsqcup F_{n c}\left(\mathbb{C} P^{m}, 3\right),
$$

where $F_{c}\left(\mathbb{C} P^{m}, 3\right)$ is the space of all configurations of three collinear points in $\mathbb{C} P^{m}$ and $F_{n c}\left(\mathbb{C} P^{m}, 3\right)$ is the configuration space of three non-collinear points. We compute the Betti numbers and (partially) the multiplicative structure of the cohomology algebras of these spaces. We begin with the finite dimensional case.

Proposition 6.1. The Poincaré polynomial of the space of configurations of three collinear points in $\mathbb{C} P^{m}$ is given by:

$$
P_{F_{C}\left(\mathbb{C} P^{m}, 3\right)}(t)=\left(1+t^{2 m+1}\right) C_{m-1}(t) .
$$


Proof. There are two natural fibrations (see $[\mathrm{FN}]$ and $[\mathrm{BP}$, Proposition 2.2):

$$
\begin{array}{ll}
(I) & S^{1} \simeq \mathbb{C} P^{1} \hookrightarrow F_{c}\left(\mathbb{C} P^{m}, 3\right) \stackrel{p}{\rightarrow} F\left(\mathbb{C} P^{m}, 2\right) \\
(I I) & F\left(\mathbb{C} P^{1}, 3\right) \hookrightarrow F_{c}\left(\mathbb{C} P^{m}, 3\right) \rightarrow G r_{1}\left(\mathbb{C} P^{m}\right)
\end{array}
$$

The bases of these fibrations are simply connected and the $\mathbf{E}_{2}$ page of the LeraySerre spectral sequence are given in the figure:
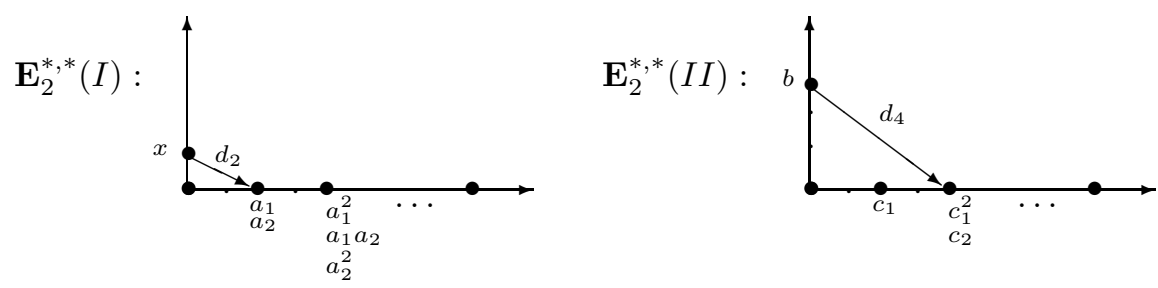

The cohomology algebra $H^{+}\left(F\left(\mathbb{C} P^{1}, 3\right)\right)$ is concentrated in degree three and the odd cohomology of the Grassmanian $G r_{1}\left(\mathbb{C} P^{m}\right)=G r_{2}\left(\mathbb{C}^{m+1}\right)$ is zero, hence $\beta_{1}\left(F_{c}\left(\mathbb{C} P^{m}, 3\right)\right)=0$. This implies that $d_{2}(x)=\lambda a_{1}+\mu a_{2}$ in $\mathbf{E}_{2}^{*, *}(I)$, where at least one of the scalars $\lambda$ and $\mu$ is non zero. By the same argument as that in Proposition 4.2 we see that $d_{2}: \mathbf{E}_{2}^{i, 1} \rightarrow \mathbf{E}_{2}^{i+2,0}$ is injective up to $i=2 m-2$ and surjective for $i=2 m-2, \ldots, 4 m-2$. Thus the Poincaré polynomial is

$$
P_{F_{c}\left(\mathbb{C} P^{m}, 3\right)}(t)=\left(1+t^{2 m+1}\right) C_{m-1}(t) \text {. }
$$

Proposition 6.2. The Poincaré polynomial of $F_{n c}\left(\mathbb{C} P^{m}, 3\right)$ is given by:

$$
P_{F_{n c}\left(\mathbb{C} P^{m}, 3\right)}(t)=C_{m-2}(t) \cdot C_{m-1}(t) \cdot C_{m}(t) .
$$

Proof. By Poincaré-Lefschetz duality we have

$$
H_{i}\left(\mathbb{C} P^{m} \backslash \mathbb{C} P^{1}\right) \cong H^{2 m-i}\left(\mathbb{C} P^{m}, \mathbb{C} P^{1}\right) ;
$$

from the cohomology long exact sequence of the pair $\left(\mathbb{C} P^{m}, \mathbb{C} P^{1}\right)$ we find

$$
P_{\mathbb{C} P^{m}} \backslash \mathbb{C} P^{1}(t)=C_{m-2}(t) .
$$

Therefore the spectral sequence associated to the fibration (see [BP], Remark 2.5)

$$
\mathbb{C} P^{m} \backslash \mathbb{C} P^{1} \hookrightarrow F_{n c}\left(\mathbb{C} P^{m}, 3\right) \rightarrow F\left(\mathbb{C} P^{m}, 2\right)
$$

is concentrated in even degrees and degenerates at the $\mathbf{E}_{2}$ term; the Poincaré polynomial is given by

$$
P_{F_{n c}\left(\mathbb{C} P^{m}, 3\right)}=C_{m-2}(t) \cdot C_{m-1}(t) \cdot C_{m}(t) .
$$

In the second part we compute the cohomology algebras for collinear and noncollinear configurations in the infinite dimensional projective space.

Proposition 6.3. The configuration space of three collinear points in $\mathbb{C} P^{\infty}$ has the cohomology algebra of $\mathbb{C} P^{\infty}$

$$
H^{*}\left(F_{c}\left(\mathbb{C} P^{\infty}, 3\right)\right) \cong H^{*}\left(\mathbb{C} P^{\infty}\right) .
$$


Proof. We will use the spectral sequence argument given in Proposition 6.1 first proving that the following are Serre fibrations:

$$
\begin{aligned}
(I) & S^{1} \simeq \mathbb{C} P^{1} \hookrightarrow F_{c}\left(\mathbb{C} P^{\infty}, 3\right) \stackrel{p}{\rightarrow} F\left(\mathbb{C} P^{\infty}, 2\right) \simeq\left(\mathbb{C} P^{\infty}\right)^{2} \\
(I I) & F\left(\mathbb{C} P^{1}, 3\right) \hookrightarrow F_{c}\left(\mathbb{C} P^{\infty}, 3\right) \rightarrow G r_{1}\left(\mathbb{C} P^{\infty}\right) .
\end{aligned}
$$

For the second one the image of $H: e^{m} \times I \rightarrow G r_{1}\left(\mathbb{C} P^{\infty}\right)$ is contained in some finite skeleton $G r_{1}\left(\mathbb{C} P^{r}\right)$. Also there is an $s$ such that $h\left(e^{m}\right) \subset\left(\mathbb{C} P^{s}\right)^{3}$ so $\operatorname{Im}(h)$ lies in $F_{c}\left(\mathbb{C} P^{\infty}, 3\right) \bigcap\left(\mathbb{C} P^{s}\right)^{3}=F_{c}\left(\mathbb{C} P^{s}, 3\right)$ :

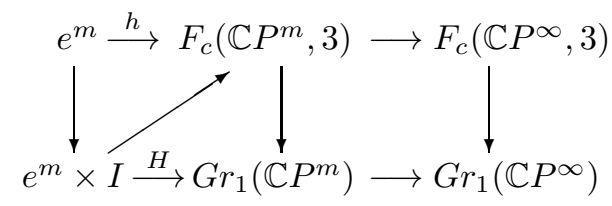

Take $m$ to be the maximum of $r$ and $s$, then the homotopy $H$ lifts to $F_{c}\left(\mathbb{C} P^{m}, 3\right)$ because $F\left(\mathbb{C} P^{1}, 3\right) \hookrightarrow F_{c}\left(\mathbb{C} P^{m}, 3\right) \rightarrow G r_{1}\left(\mathbb{C} P^{m}\right)$ is a locally trivial fibration (see $[\mathrm{BP}]$ ). Similarly one can show that (II) is also a Serre fibration. The spectral sequences associated to these fibrations start with:
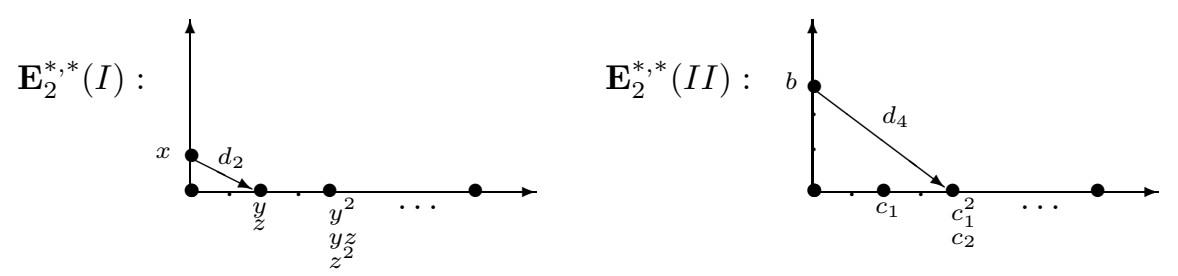

From the second spectral sequence, the first Betti number of $F_{c}\left(\mathbb{C} P^{\infty}, 3\right)$ is zero, hence in the first spectral sequence we have $d_{2}(x)=y$, after a change of basis. Therefore, in the first spectral sequence,

$$
\mathbf{E}_{3} \cong \mathbf{E}_{\infty} \cong \mathbb{C}[z] \text { where }|z|=2 .
$$

Remark 6.4. Using the ideas from Theorem 5.1 one can find a map $f$

$$
\mathbb{C} P^{\infty} \stackrel{f}{\rightarrow} F_{c}\left(\mathbb{C} P^{\infty}, 3\right) \stackrel{p r_{1}}{\rightarrow} \mathbb{C} P^{\infty}, P=\left[p_{0}: p_{1}: \ldots\right] \mapsto\left(Q_{0}, Q_{1}, Q_{2}\right),
$$

where $Q_{0}=\left[p_{0}: 0: p_{1}: 0: \ldots\right], Q_{1}=\left[0: p_{0}: 0: p_{1}: \ldots\right], Q_{2}=\left[p_{0}: p_{0}: p_{1}: p_{1}:\right.$ ...], such that $p r_{1} \circ f \simeq i d$, hence the algebraic isomorphism from Proposition 6.3 is induced by a continuous map.

Proposition 6.5. The configuration space of three non-collinear points in $\mathbb{C} P^{\infty}$ has the homotopy type of the ordered configuration space of three points

$$
F_{n c}\left(\mathbb{C} P^{\infty}, 3\right) \simeq F\left(\mathbb{C} P^{\infty}, 3\right) \simeq\left(\mathbb{C} P^{\infty}\right)^{3} .
$$

Proof. In the proof of Theorem [5.1, the points $Q_{0}, Q_{1}, Q_{2}$ are non-collinear.

Remark 6.6. More generally, $F_{n c}\left(\mathbb{C} P^{\infty}, n\right) \simeq\left(\mathbb{C} P^{\infty}\right)^{n}$, with the same proof. 


\section{Appendix: Counting PARTitions in three PARTS}

In this elementary section we give closed formulae for the number of partitions

$$
\begin{aligned}
P_{3}(k) & =\operatorname{card}\{(a, b, c) \mid a \geq b \geq c \geq 0, a+b+c=k\}, \\
P_{3, \leq m}(k) & =\operatorname{card}\{(a, b, c) \mid m \geq a \geq b \geq c \geq 0, a+b+c=k\}
\end{aligned}
$$

and we prove unimodality for the sequences of even and odd Betti numbers for the configuration spaces discussed in this paper. We will denote by $\lfloor x\rceil$ the nearest integer to the real number $x$; for $x \in \mathbb{Z}+\frac{1}{2}$ this is not defined but in all the following formulae this never happens.

Proposition 7.1. a) The number of positive partitions of $k$ into three parts a+ $b+c=k, a \geq b \geq c \geq 1$ is given by $\left\lfloor\frac{k^{2}}{12}\right\rceil$.

b) The number of non-negative partitions of $k$ is given by $P_{3}(k)=\left\lfloor\frac{(k+3)^{2}}{12}\right\rceil$

Proof. a) An expanded version for this (six cases) is given in [H]. The closed form can be found in $\mathrm{YY}$.

b) The non-negative partitions $(a, b, c)$ of $k$ are in bijection with positive partitions $(a+1, b+1, c+1)$ of $k+3$.

Proposition 7.2. The number of bounded partitions $P_{3, \leq m}(k)$ satisfy the following properties:

a) For $0 \leq k \leq m, P_{3, \leq m}(k)=P_{3}(k)=\left\lfloor\frac{(k+3)^{2}}{12}\right\rceil$.

b) For $k \geq 3 m+1, P_{3, \leq m}(k)=0$.

c) For $\frac{3 m}{2} \leq k \leq 3 m, P_{3, \leq m}(k)=P_{3, \leq m}(3 m-k)$.

Proof. a) and b) are obvious, and for c) use the bijection

$$
(a, b, c) \leftrightarrow(m-c, m-b, m-a)
$$

From this result, it is sufficient to compute the value of $P_{3, \leq m}(k)$ for $k$ between $m+1$ and $\left\lfloor\frac{3 m}{2}\right\rfloor$.

Proposition 7.3. For $m+1 \leq k \leq\left\lfloor\frac{3 m}{2}\right\rfloor$, the number of bounded partitions is given by

$$
P_{3, \leq m}(k)=\left\lfloor\frac{(k+3)^{2}}{12}\right\rceil-\left\lfloor\frac{(k-m-1)^{2}}{4}\right\rceil+m-k .
$$

Proof. From the total number of non-negative partitions, $P_{3}(k)$, the number of solutions of $a+b+c=k$ satisfying $\max (a, b, c) \geq m+1$ should be subtracted. Because $a \geq b \geq c$ and $k \leq\left\lfloor\frac{3 m}{2}\right\rfloor$, only $a$ could be greater than $m: a=m+1, m+$ $2, \ldots, k$. For a fixed $a$, the number of solutions of

$$
b+c=k-a, \quad b \geq c \geq 0
$$

equals $\left\lfloor\frac{k-a}{2}\right\rfloor+1$. A simple computation gives $\left\lfloor\frac{0}{2}\right\rfloor+\left\lfloor\frac{1}{2}\right\rfloor+\ldots+\left\lfloor\frac{p}{2}\right\rfloor=\left\lfloor\frac{p^{2}}{4}\right\rfloor$, and taking $p=k-m-1$, we obtain the formula.

Now we show that the sequences of even and odd Betti numbers of the cohomology algebra $H^{*}\left(C\left(\mathbb{C} P^{m}, 3\right)\right) \cong H^{*}\left(F\left(\mathbb{C} P^{m}, 3\right)\right)(3)$ are unimodal.

Lemma 7.4. a) The sequence $\left(P_{3}(k)\right)_{k \geq 0}$ is increasing (strictly for $k \geq 1$ ).

b) The sequence $\left(P_{3, \leq m}(k)\right)_{0 \leq k \leq 3 m}$ is unimodal and symmetric, more precisely: $1=P_{3, \leq m}(0)=P_{3, \leq m}(1)<P_{3, \leq m}(2)<\ldots<P_{3, \leq m}\left(\left\lfloor\frac{3 m}{2}\right\rfloor\right)=$

$=P_{3, \leq m}\left(\left\lceil\frac{3 m}{2}\right\rceil\right)>\ldots>P_{3, \leq m}(3 m-1)=P_{3, \leq m}(3 m)=1$ 
Proof. a) A solution of the equation $a+b+c=k$ gives the solution $(a+1)+b+c=$ $k+1$ and solutions of the form $a+a+b=k+1(a \geq b)$ can not be obtained in this way (for $k \neq 1$ one can find solutions of the form $a+a+b=k+1$ ).

b) Similarly, for $k \leq\left\lfloor\frac{3 m}{2}\right\rfloor$, a solution $a+b+c=k, m-1 \geq a \geq b \geq c$, gives a solution $(a+1)+b+c=k+1, m \geq a+1 \geq b \geq c$, and a solution of the form $m+b+c=k$ gives a solution $m+(b+1)+c=k+1$ ( $b$ can not be equal to $m$ for $\left.k \leq\left\lfloor\frac{m}{2}\right\rfloor\right)$. Again we have strict inequality for $k \geq 1$ because solutions of the form $a+a+b=k,(a \geq b)$, are not in the image of the previous injective map.

Proof of Corollary 1.2. Case 1: Betti numbers of $F\left(\mathbb{C} P^{m}, 3\right)$. The even Poincaré polynomial equals $\left(1+t^{2}+\ldots+t^{2 m-1}\right)^{3}$ and the odd Poincaré polynomial equals $t^{4 m-1}\left(1+t^{2}+t^{4}+\ldots+t^{2 m-2}\right)$.

Case 2: $V(3)$-Betti numbers. The sequence of odd Betti numbers is $\beta_{1}=\beta_{3}=$ $\ldots=\beta_{4 m-3}=0, \beta_{4 m-1}=\beta_{4 m+1}=\ldots=\beta_{6 m-3}=1$. The sequence of even Betti numbers can be splitted into four parts:

i) If $0 \leq k \leq m-1, \beta_{2 k}=P_{3, \leq m}(k)$ : the sequence is increasing (strictly increasing for $k \geq 1$ );

ii) If $m \leq k \leq\left\lfloor\frac{3 m}{2}\right\rfloor, \beta_{2 k}=P_{3, \leq m}(k)+m-k-1$ : the sequence is increasing because $P_{3, \leq m}(k)<P_{3, \leq m}(k+1)$ implies

$$
P_{3, \leq m}(k)+m-k-1 \leq P_{3, \leq m}(k+1)+m-k-2 ;
$$

iii) If $\left\lceil\frac{3 m}{2}\right\rceil \leq k \leq 2 m-1, \beta_{2 k}=P_{3, \leq m}(k)-3 m+k$ : the sequence is the sum of two strictly decreasing sequences;

iv) If $2 m \leq k \leq 3 m-1, \beta_{2 k}=P_{3, \leq m}-3 m+k$ : the sequence is decreasing as in the second case.

Finally, the join of these sequences is unimodal:

a) $\beta_{2 m-2} \leq \beta_{2 m}$, because $P_{3, \leq m}(m-1) \leq P_{3, \leq m}(m)-1$ (here we need $m \geq 2$ )

b) $\beta_{2\left\lfloor\frac{3 m}{2}\right\rfloor} \geq \beta_{2\left\lceil\frac{3 m}{2}\right\rceil} ; P_{3, \leq m}\left(\left\lfloor\frac{3 m}{2}\right\rfloor\right)+m-\left\lfloor\frac{3 m}{2}\right\rfloor-1 \geq P_{3, \leq m}\left(\left\lceil\frac{3 m}{2}\right\rceil\right)+m-\left\lceil\frac{3 m}{2}\right\rceil-1$;

c) $\beta_{4 m-2} \geq \beta_{4 m}$, because $P_{3, \leq m}(2 m-1)-m \geq P_{3, \leq m}(2 m)-m$.

Case 3: $V(2,1)$-Betti numbers. Like in the previous case, the terms of the first two sums in the Proposition 3.12 are given by an increasing sequence and those of the last two sums are given by a decreasing sequence.

Case 4: $V(1,1,1)$-Betti numbers. Using Proposition 3.14this is obvious: the even sequence starts with three zeros, next is the increasing sequence $P_{3, \leq m-2}(k-3)$, $3 \leq k \leq\left\lfloor\frac{3 m}{2}\right\rfloor$, next the symmetric decreasing sequence, at the end there are three zeros and the odd sequence is constant zero.

Remarks 7.5. a) In the special case of the projective line $(m=1)$, the sequences of Betti numbers are still unimodal:

$$
P_{C\left(\mathbb{C} P^{1}, 3\right)}(t)=1+t^{3} .
$$

b) The sequences of even Betti numbers is not symmetric, for instance

$$
P_{C\left(\mathbb{C} P^{2}, 3\right)}(t)=\left(1+t^{2}+2 t^{4}+t^{6}\right)+\left(t^{7}+t^{9}\right) .
$$

\section{REFERENCES}

[A] V.I. Arnold, The cohomology ring of dyed braids, Mat. Zametki 5 (1969), 227-231.

[AAB] S. Ashraf, H. Azam, B. Berceanu, Representation theory for the Križ model, arXiv: 1106.4926v1 [math.RT] (2012).

[BMP] B. Berceanu, M. Markl, S. Papadima, Multiplicative models for configuration spaces of algebraic varieties, Topology 44 (2005), 415-440. 
[BP] B. Berceanu, S. Parveen, Braid groups in complex projective spaces, Advances in Geometry 12 (2012), 269-286.

[B] R. Bezrukavnikov, Koszul dg-algebras arising from configuration spaces, Geometric and Functional Analysis 4(2) (1994), 119-135.

[CT] F. Cohen, L. Taylor, Computations of Gelfand-Fuks cohomology, the cohomology of function spaces, and the cohomology of configuration spaces; in: Geometric Applications of Homotopy Theory I, Proceedings, Evanston 1977, Lecture Notes in Mathematics vol. 657, 106-143, Springer-Verlag 1978.

[FaH] E.R. Fadell, S.Y. Husseini, Geometry and Topology of Configuration Spaces, Springer Monographs in Mathematics, Springer-Verlag, Berlin, 2001.

[FN] E. Fadell, L. Neuwirth, Configuration spaces, Math. Scand. 10 (1962), 111-118.

[FZ] E. M. Feichtner, G. M. Ziegler, The integral cohomology algebras of ordered configuration spaces of spheres, Documenta Mathematica 5 (2000), 115-139.

[FOT] Y. Felix, J. Oprea, D. Tanré, Algebraic Models in Geometry, Oxford Graduate Texts in Mathematics 17, 2008.

[FT] Y. Felix, D. Tanré, The cohomology algebra of unordered configuration spaces, J. of London Math. Soc. 72(2) (2005), 525-544.

[FTh] Y. Felix, J. C. Thomas, Rational Betti numbers of configuration spaces, Topology Appl. 102(2) (2000), 139-149.

[FH] W. Fulton, J. Harris, A First Course in Representation Theory, Graduate Texts in Mathematics 129, Springer-Verlag Berlin, 1991.

[FM] W. Fulton, R. MacPherson, A compactification of configuration spaces, Ann. of Math. 139 (1994), 183-225.

[H] M. Hall, Jr., Combinatorial Theory, Wiley Interscience, 1998.

[K] I. Kriz, On the rational homotopy type of configuration spaces, Ann. of Math. 139 (1994), 227-237.

[LS] G. I. Lehrer, L. Solomon, On the action of the symmetric group on the cohomology of the complement of its reflecting hyperplanes, J. Algebra 104 (1986), 410-424.

[S] T. Sohail, Cohomology of configuration spaces of complex projective spaces, Czechoslovak Math. Journal vol. 60(2) (2010), 411-422.

[T] B. Totaro, Configuration spaces of algebraic varieties, Topology 35(4) (1996), 1057-1067.

[YY] A. M. Yaglom, I. M. Yaglom, Challenging Mathematical Problems with Elementary Solutions, Holden-Day, Inc., San Francisco, CA, 1967.

${ }^{1}$ Abdus Salam School of Mathematical Sciences, GC University, Lahore-Pakistan.

E-mail address: samia.ashraf@yahoo.com

${ }^{2}$ Abdus Salam School of Mathematical Sciences, GC University, Lahore-Pakistan, and Institute of Mathematics Simion Stoilow, Bucharest-Romania (Permanent address).

E-mail address: Barbu.Berceanu@imar.ro 